\title{
A CHECKLIST OF REPTILES OF KERALA, INDIA
}

\author{
Muhamed Jafer Palot
}

ISSN $0974-7907$ (Online) ISSN 0974-7893 (Print)
Zoological Survey of India (ZSI), Western Ghat Regional Centre, Jafer Khan Colony, Eranhipalam, Kozhikode, Kerala 673006, India palot.zsi@gmail.com

\section{OPEN ACCESS}

Abstract: A checklist of reptiles of Kerala State is presented, along with their Scientific, English and Malayalam names, endemic status, conservation status in the latest IUCN Red List category, different Schedules of Indian Wildlife (Protection) Act and the Appendices of CITES. A total of 173 species under 24 families belonging to three orders are recorded from Kerala. Of these, 87 species are endemic to the Western Ghats, which include the 10 Kerala endemics. Of the 173 species, 23 are listed in the various threatened categories of IUCN.

Keywords: CITES, Indian Wildlife (Protection) Act, Malayalam name, vernacular name, Western Ghats.

Research on reptiles in India dates back to more than 200 years, coinciding with the formation of the erstwhile British East India Company. The monumental works on Indian reptiles are Catalogue of the Reptiles inhabiting the Peninsular of India (Jerdon 1853), The Reptiles of British India (Gunther 1864), Fauna of British India Reptilia and Batrachia (Boulenger 1890) and the three volume series of The Fauna of British India including Ceylon and Burma, including the whole of Indo-Chinese sub-region (Smith 1931, 1935, 1943). The work of Smith stood the test of time and forms the standard work on this subject including the Kerala region. Since the publications of above cited works, our knowledge on the reptile fauna of the region has been enriched further by the publications of Sharma (1998, 2002, 2007), Inger et al. (1984), Rajendran (1985), Murthy (1981, 1985), Tikader \& Sharma (1992), Das (1997, 2002, 2003), Molur \& Walker (1998), Daniel (2002), Whitaker \& Captain (2004), Vogel \& Van Rooijen (2009), Vasudevan (2009), Chandramouli \& Ganesh (2010), Venugopal (2010), Ganesh \& Chandramouli (2013), Srinivasulu et al. (2014) and Ganesh et al. (2014). Altogether 518 species of reptiles have been reported from India (Aengals et al. 2011). A few more species have been reported from India since then (Smith et al. 2012; Harikrishnan et al. 2013; Aengals \& Ganesh 2013; Vogel \& Ganesh 2013; Cyriac \& Umesh 2014; Mirza \& Sanap 2014; Mirza et al. 2014; Amarasinghe et al. 2015; Guptha et al. 2015; Ágarwal \& Karanth 2015).

In Kerala, reptiles comprise poorly studied classes of vertebrates. Though there have been a few attempts to study reptiles in Kerala (Beddome 1878, 1886; Boulenger
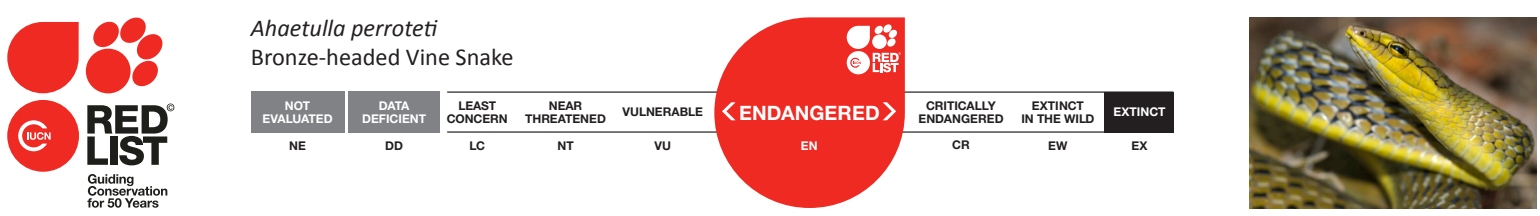

DOI: http://dx.doi.org/10.11609/jott.2002.7.13.8010-8022 | ZooBank: urn:Isid:zoobank.org:pub:FF9AD693-3492-445A-B183-52DEE350F619

Editor: Mewa Singh, University of Mysore, Mysuru, India.

Date of publication: 17 November 2015 (online \& print)

Manuscript details: Ms \# 04306 | Received 11 May 2015 | Final received 23 September 2015 | Finally accepted 29 September 2015

Citation: Palot, M.J. (2015). A checklist of reptiles of Kerala, India. Journal of Threatened Taxa 7(13): 8010-8022; http://dx.doi.org/10.11609/jott.2002.7.13.8010-8022

Copyright: (C) Palot 2015. Creative Commons Attribution 4.0 International License. JoTT allows unrestricted use of this article in any medium, reproduction and distribution by providing adequate credit to the authors and the source of publication.

Funding: None.

Conflict of Interest: The author declares no competing interests.

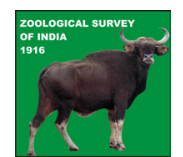

Acknowledgements: The author is grateful to the Director, Zoological Survey of India, Kolkata and the Officer-in-Charge, Zoological Survey of India, Kozhikode for facilities and encouragements. I would also like to acknowledge the contribution of my team members viz., PO Nameer, Praveen J, Rajeev Raghavan, A Bijukumar and Sandeep Das in getting this checklist into a final shape. Sandeep Das contributed the images used along with this paper. 
1890; Ferguson 1895; Wall 1905, 1918; Murthy 1981; Inger et al. 1984; Radhakrishnan 1996, 1997; Thomas et al. 1997; Thomas \& Easa 1997; Joseph \& Easa 1997; Zacharias 1997; Easa 1998; Abraham et al. 1999; Ajith 2000; Palot \& Radhakrishnan 2002, 2003, 2004; Chandramouli \& Ganesh 2010; Cyriac et al. 2011; Jins et al. 2014). A book on the Snakes of Kerala in Malayalam by Adiyodi (1965) is the first published work pertaining to the snakes of the state. The first comprehensive checklist on the reptilian fauna of the State was compiled by Radhakrishnan (1997) who listed 169 species. Subsequently, Palot \& Radhakrishnan (2003) compiled a checklist of vernacular names of snakes of Kerala. Further, as part of biodiversity documentation, the Kerala Forest Research Institute (KFRI) published a series of checklists on the biodiversity of the State including reptiles (Easa \& Ramachandran 2004). More recently, Palot \& Radhakrishnan (2011) updated the checklist of reptiles of Kerala by listing 173 species from the region.

In recent years, there has been a revival of interest in research on the reptilian fauna of the State, with several new species' descriptions from the region (Manamendra-Aracchi et al. 2007; Gower \& Winkler 2007; Vogel \& van Rooijen 2011; Cyriac \& Umesh 2014). For the compilation of this checklist, the works of Smith (1931, 1935, 1943), Tikader \& Shrama (1992), Daniel (2002), Das (2002), Whitaker \& Captain (2004), Venugopal (2010) and Aengals et al. (2011) were also referred to.

The current checklist of Kerala are represented by 173 species in 24 families under three orders (Table 1). Of these, 87 species are endemic to the Western Ghats, which include 10 species endemic to Kerala. The taxonomy and nomenclature follow Uetz \& Hosek (2015) while the vernacular names have been taken from various sources (Adiyodi 1965; Radhakrishnan 1997; Palot \& Radhakrishnan 2004; 2011). Marine reptiles in the list are indicated in blue colour.

\section{REFERENCES}

Abraham, S., P.S. Easa, J. Thomas \& M.A. Nixon (1999). An inventory of reptile fauna in Chinnar Wildlife Sanctuary, Idukki District, Kerala. Cobra 37: 24-29.

Adiyodi, K.G. (1965). Keralathile Vishapampukal. (in Malayalam). The Mathrubhumi Printing \& Publishing Co., Ltd. Kozhikode, Kerala, India.

Aengals, R. \& S.R. Ganesh (2013). Rhinophis goweri - a new species of Shieldtail snake from the southern Eastern Ghats, India. Russian Journal of Herpetology 20(1): 61-65.

Aengals, R., V.M.S. Kumar \& M.J. Palot (2011). Updated Checklist of Indian Reptiles. http://zsi.gov.in/checklist/Checklist\%20of\%20 Indian\%20Reptiles.pdf (online version).
Agarwal, I. \& K.P. Karanth (2015). A phylogeny of the only grounddwelling radiation of Cyrtodactylus (Squamata, Gekkonidae): diversification of Geckoella across peninsular India and Sri Lanka. Molecular Phylogenetics and Evolution 82(part-A): 193-199; http:dx.doi.org/10.1016/j.ympev.2014.09.016

Ajit, V.P. (2000). Rediscovery of two rare Typhlopoids, Typhlops thurstoni Boettger, 1890 and T. tindalli Smith, 1943 from Kerala. Journal of the Bombay Natural History Society 97(3): 434-435.

Amarasinghe, A.A.T., I. Ineich, D.M.S.S. Karunarathna, W.M.S. Botejue \& P.D. Campbell (2015). Two new species of the genus Sitana Cuvier, 1829 (Reptilia: Agamidae) from Sri Lanka, including a taxonomic revision of the Indian Sitana species. Zootaxa 3915: 67-98; http://dx.doi.org/10.11646/zootaxa.3915.1.3

Beddome, R.H. (1886). An account of the earth-snakes of the peninsula of India and Ceylon. The Annals Magazine of Natural History (5) xvii: 3-33.

Beddome, R.H. (1878). Description of six new species of snakes of the genus Silybura, family Uropeltidae. Proceedings of the Zoological Society of London 1878: 800-802.

Boulenger, G.A. (1890). The fauna of British India including Ceylon and Burma: Reptilia and Batrachia. London, viii+541pp.

Chandramouli, S.R. (2009). Status and microhabitat preference of Otocryptis beddomii Boulenger, 1885 (Reptilia: Agamidae) in Ponmudi Hills, Western Ghats, Kerala, India. Taprobanica 1(2): 107-110.

Chandramouli, S.R \& S.R. Ganesh (2010). Herpetofauna of southern Western Ghats, India reinvestigated after decades. Taprobanica 2(2): 72-85.

Cyriac, V.P, C.P. Arjun \& T.K. Joy (2011). Occurrence of the Anamalai Gecko Hemidactylus anamallensis Gunther, 1875 from Chembra, Wayanad, Kerala. Malabar Trogon 9(3): 20-21.

Cyriac, V.P. \& P.K. Umesh (2014). Description of a New GroundDwelling Cnemaspis Strauch, 1887 (Squamata: Gekkonidae), from Kerala, allied to C. wynadensis (Beddome, 1870). Russian Journal of Herpetology 21(3): 187-194.

Daniel, J.C. (2002). The Book of Indian Reptiles. Bombay Natural History Society, Bombay, 141pp.

Das, I. (1997). Checklist of the reptiles of India with English common name. Hamadryad 22: 32-45.

Das, I. (2002). A Photographic Guide to the Snakes and Other Reptiles of India. New Holland Publishers (U.K.) Ltd., London, 144pp.

Das, I. (2003). Growth of Knowledge on the Reptiles of India, with an Introduction to Systematics, Taxonomy and Nomenclature. Journal of Bombay Natural History Society 100(2-3): 447-501.

Easa, P.S. (1998). Survey of Reptiles and Amphibians in Kerala part of Nilgiri Biosphere Reserve. KFRI Research Report No.148. Kerala Forest Research Institute, Peechi.

Easa, P.S \& K.K. Ramachandran (2004). Biodiversity Documentation for Kerala. Part 10: Reptiles. Kerala Forest Research Institute, Peechi.

Ferguson, H.S. (1895). List of snakes taken in Travancore from 188895. Journal of the Bombay Natural History Society 10: 68-77.

Ganesh. S.R. \& S.R. Chandramouli (2013). Endangered and enigmatic reptiles of Western Ghats - an overview. In: Singaravelan, N. (ed.). Rare Animals of India, Bentham Publishers, 35-36.

Ganesh, S.R., S. Bhupathy, P. David, N. Sathishkumar \& G. Srinivas (2014). Snake fauna of High Wavy Mountains, Western Ghats, India: species richness, status, and distribution pattern. Russian Journal of Herpetology 21(1): 53-64.

Gower, D.J. \& J.D. Winkler (2007). Taxonomy of the Indian snake Xylophis Beddome (Serpentes: Caenophidia) with description of a new species. Hamadryad 31(2): 315-329.

Gunther, A.C.L.G (1864). The Reptiles of British India. Published by Oxford and IBH Publishing Co. New Delhi, 452pp.

Guptha, B., N.M. Sivaramprasad, S.T. Muddock \& V. Deepak (2015). First record of Chrysopelia taprobanica Smith, 1943 (Squamata: Colubridae) from India. Checklist 11(1): 1-3.

Harikrishnan, S., K. Vasudevan, A. De Silva, V. Deepak., N.B. Kar \& R. Naniv (2012). Phylogeography of Dasia Gray, 1830 (Reptilia: 


\begin{tabular}{|c|c|c|c|c|c|c|c|c|c|c|c|c|c|c|c|c|c|}
\hline 岂 & $\overline{\text { o }}$ & $\begin{array}{l}-\overline{0} \\
\frac{0}{8}\end{array}$ & $\begin{array}{l}\overline{\overline{0}} \\
\frac{0}{\alpha}\end{array}$ & $\begin{array}{l}= \\
\overline{0} \\
\frac{0}{\alpha}\end{array}$ & $\overline{\frac{0}{8}}$ & $\overline{\dot{0}}$ & $\overline{\dot{0}}$ & $\overline{\dot{0}}$ & $\begin{array}{l}\overline{=} \\
\frac{0}{9}\end{array}$ & $\begin{array}{l}\overline{=} \\
\frac{0}{8}\end{array}$ & $\begin{array}{l}\overline{\overline{0}} \\
\frac{0}{8}\end{array}$ & $\begin{array}{l}\overline{=} \\
\frac{0}{8}\end{array}$ & $\begin{array}{l}\overline{=} \\
\frac{\dot{0}}{\alpha}\end{array}$ & $\overline{\text { o. }}$ & & & \\
\hline$\frac{\hat{a}}{3}$ & $\overline{\vec{n}}$ & $\overline{\text { si }}$ & $\overline{\overline{\dot{s}}}$ & & $\overline{\underline{\dot{n}}}$ & $\overline{\underline{\dot{L}}}$ & $\overline{\overline{\dot{s}}}$ & $\overline{\text { s. }}$ & $\begin{array}{l}\geq \\
\geq \\
\text { i }\end{array}$ & $\begin{array}{l}\geq \\
\text { 宫 }\end{array}$ & $\begin{array}{l}\geq \\
\geq \\
\dot{\vec{\omega}} \\
\dot{\omega}\end{array}$ & $\overline{\text { 它 }}$ & $\overline{\overline{\dot{s}}}$ & 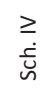 & & & \\
\hline$\sum_{u}^{0}$ & & & $\varliminf_{3}^{0}$ & & & & & & & ${ }^{\circ}$ & & & & & & $\stackrel{0}{3}$ & 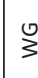 \\
\hline ב & צ & 3 & z & z & z & đ̛ & 3 & 3 & y & 3 & 3 & u & z & z & 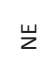 & $\breve{~}$ & $u$ \\
\hline
\end{tabular}

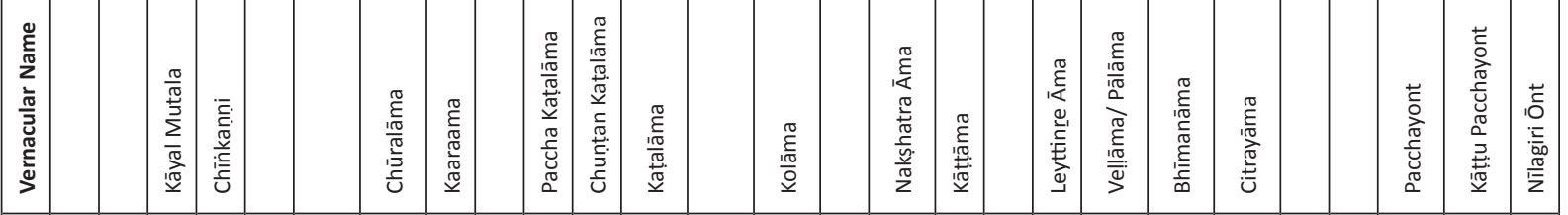

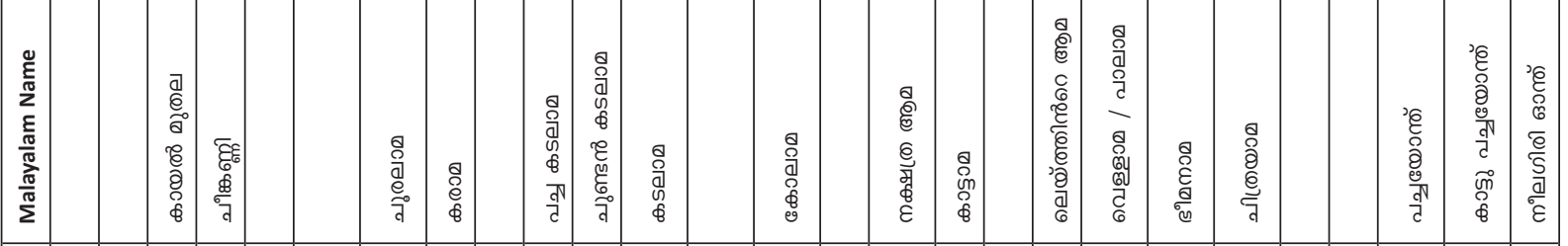

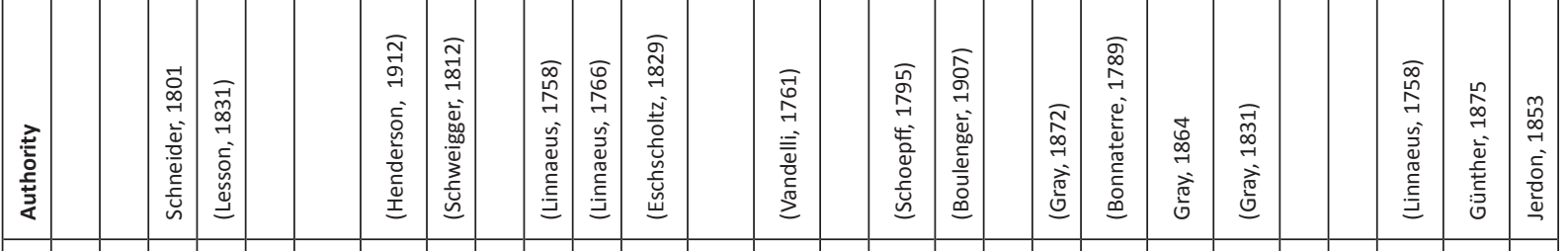

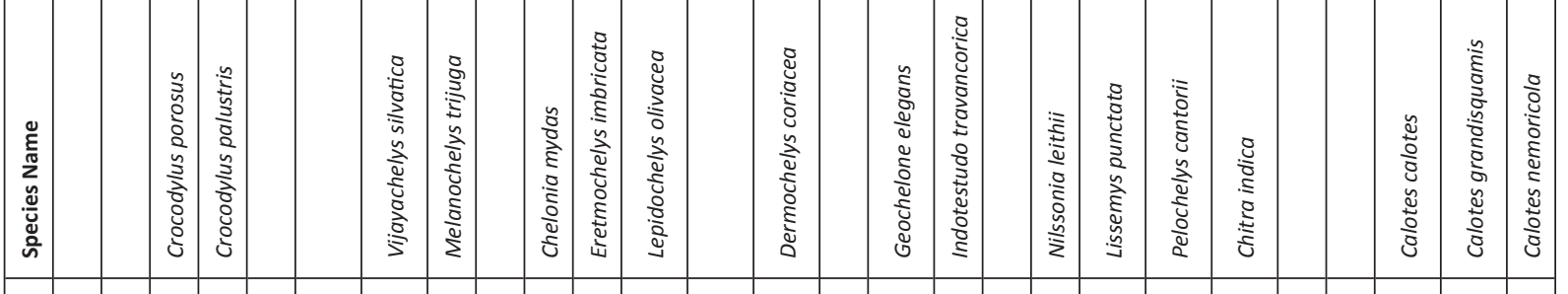

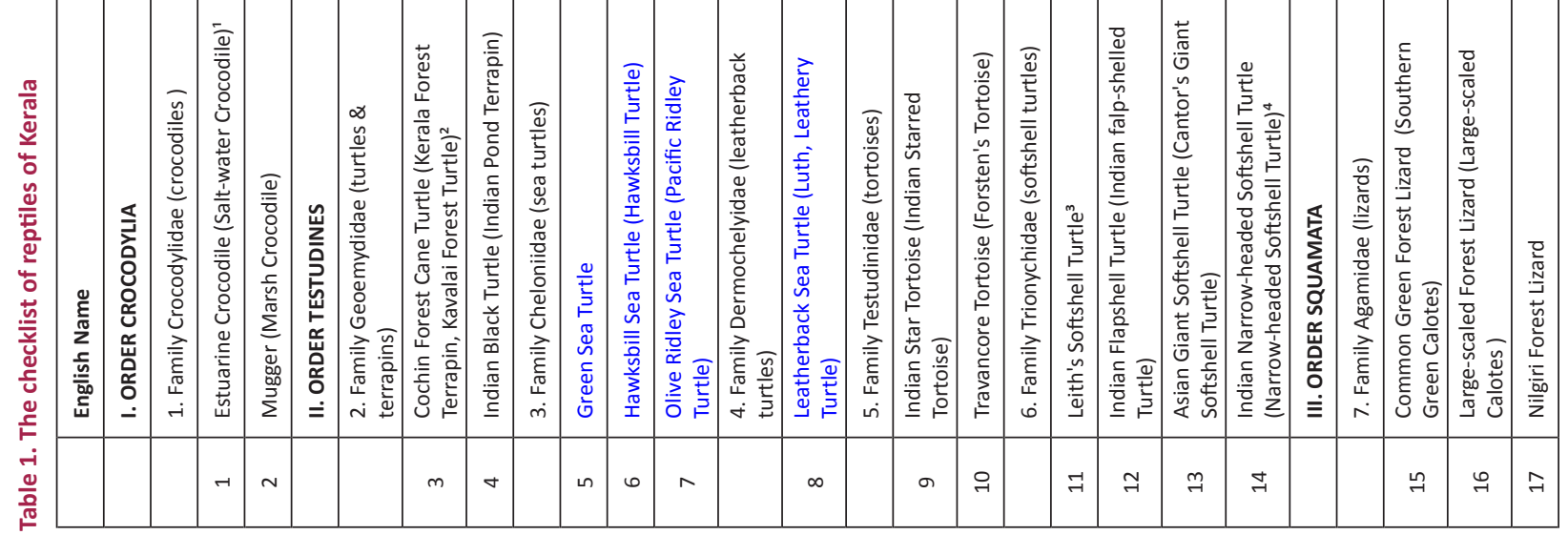




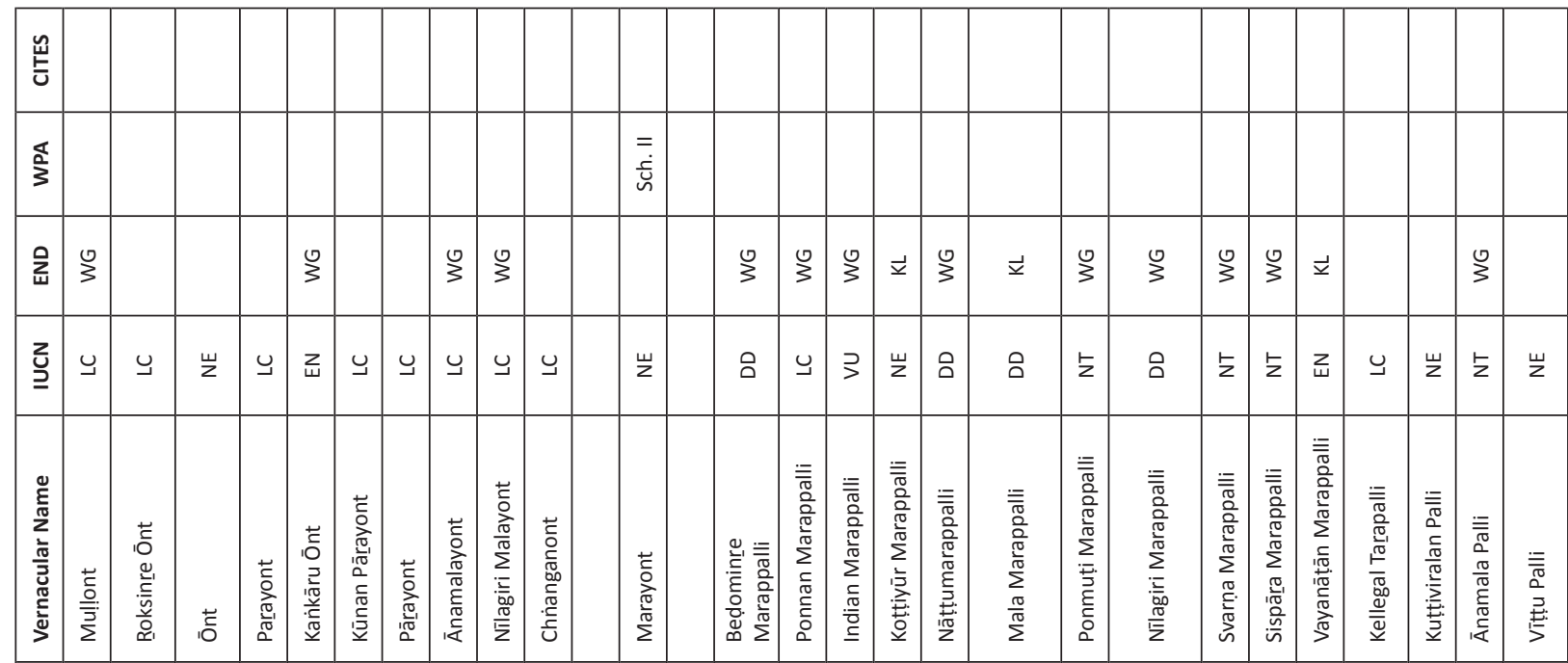

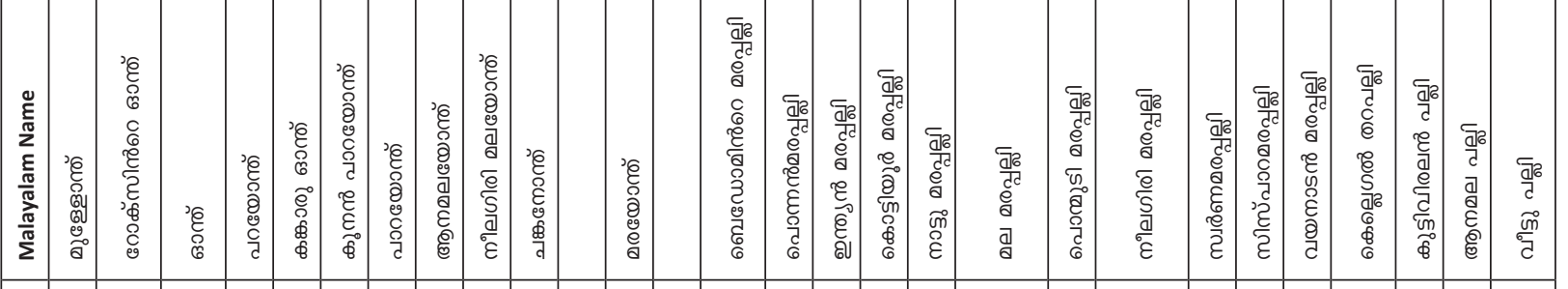

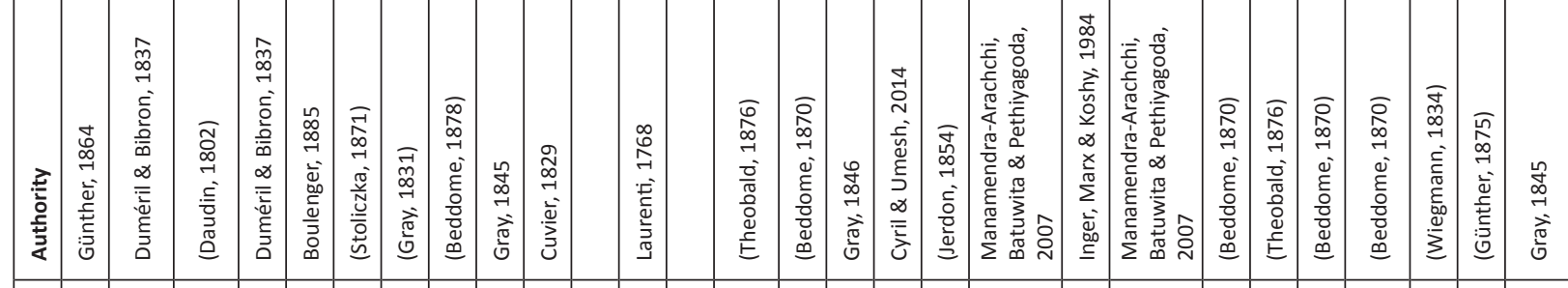

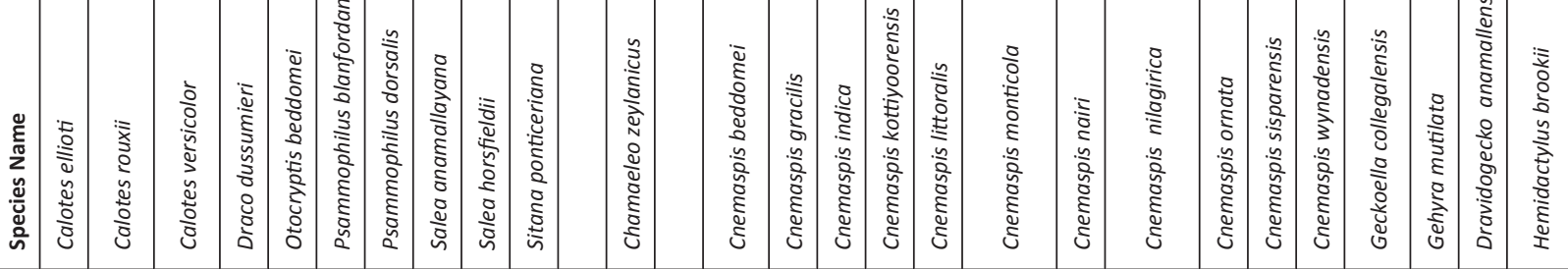

\begin{tabular}{|c|c|c|c|c|c|c|c|c|c|c|c|c|c|c|c|c|c|c|c|c|c|c|c|}
\hline & 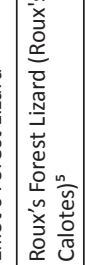 & 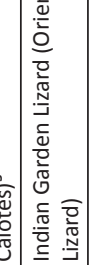 & 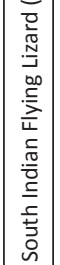 & 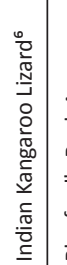 & 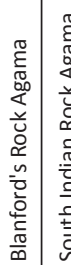 & 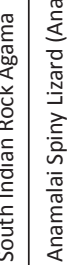 & 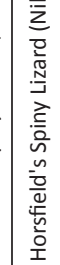 & 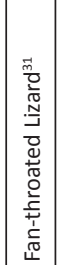 & 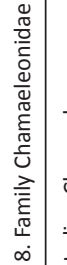 & 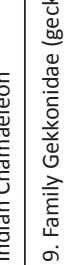 & 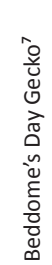 & 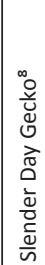 & 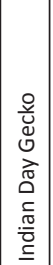 & 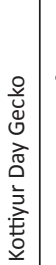 & 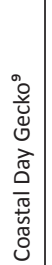 & 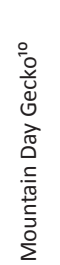 & 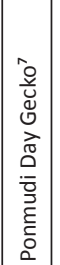 & 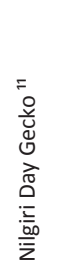 & 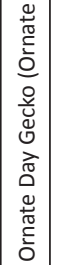 & 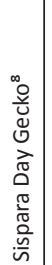 & 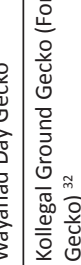 & 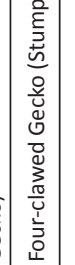 & \\
\hline & $g$ & ㄱ & $\bar{\lambda}$ & $\approx$ & $\tilde{\sim}$ & 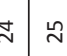 & $\stackrel{\sim}{2}$ & $\hat{\sim}$ & & & న & m & $\vec{m}$ & $\approx$ & $\stackrel{m}{m}$ & $\stackrel{m}{m}$ & $\stackrel{\sim m}{m}$ & ${ }_{n}^{\circ}$ & $\hat{m}$ & $\stackrel{\infty}{m}$ & $q$ & $\vec{\sigma}$ & \\
\hline
\end{tabular}




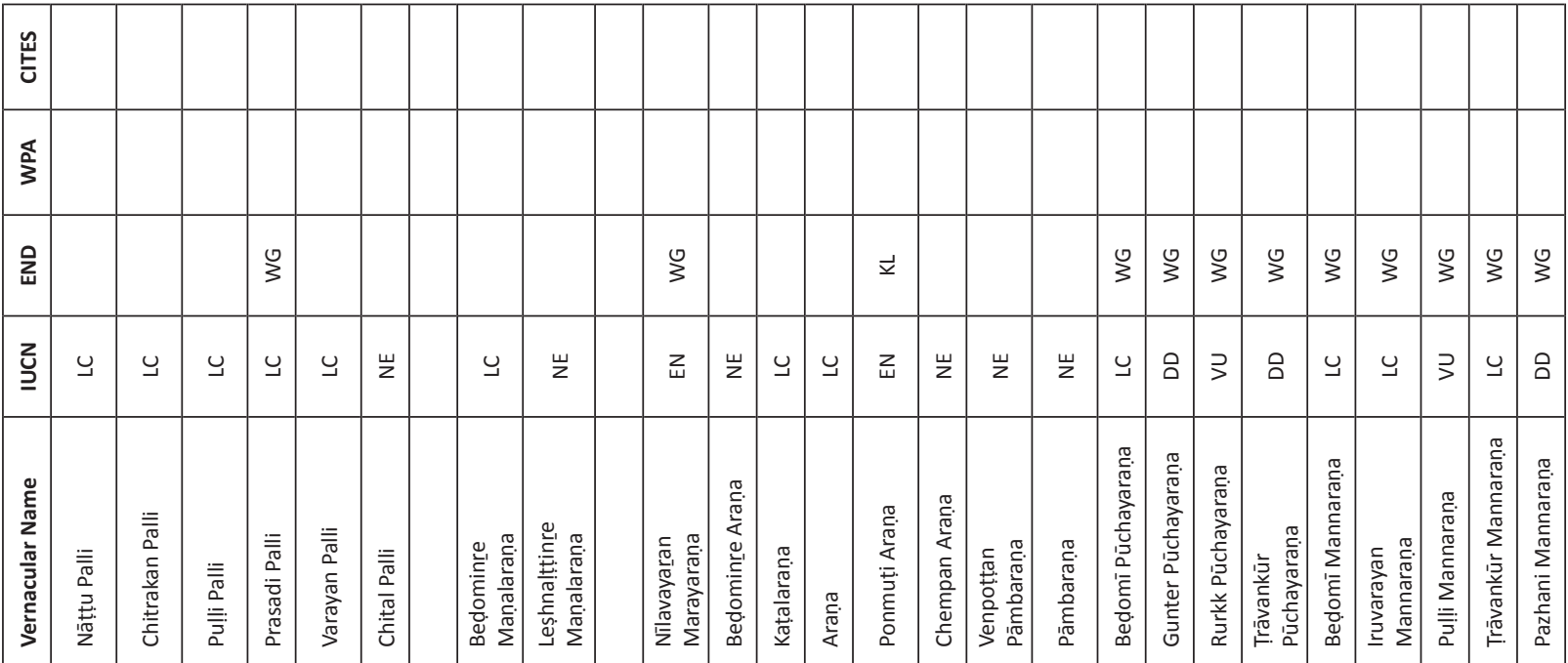

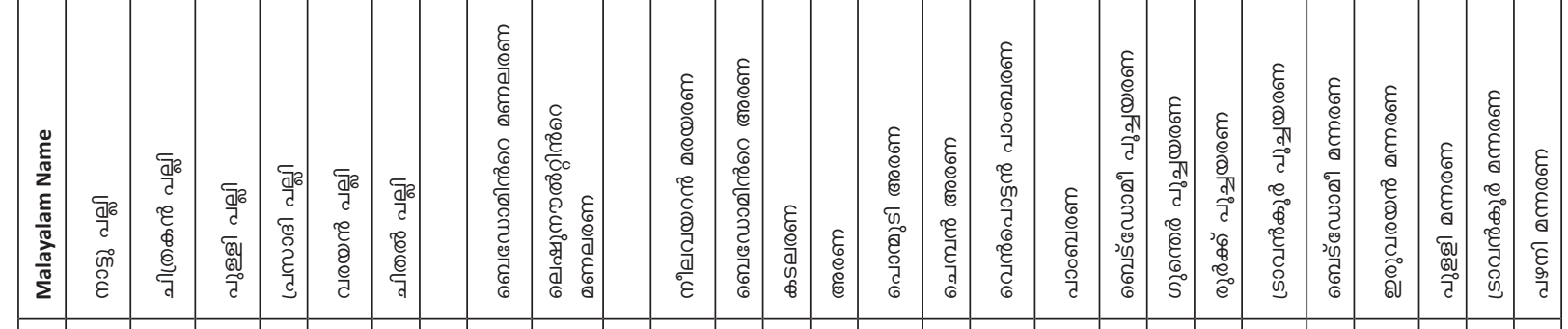

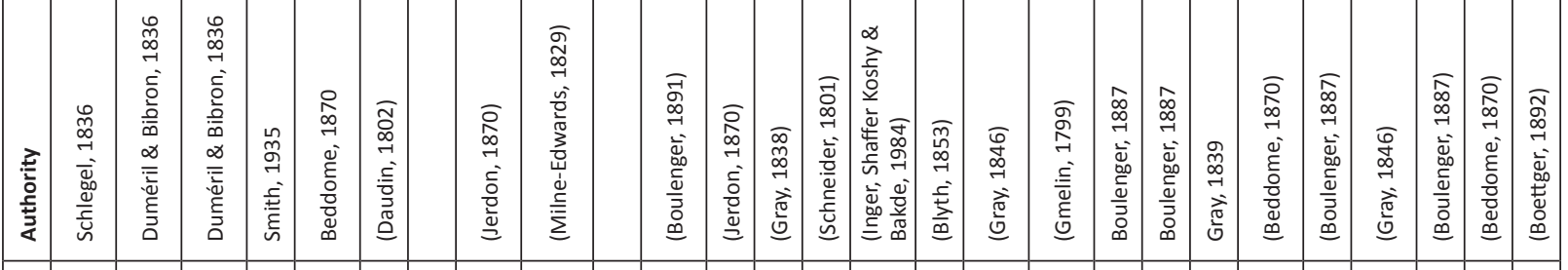

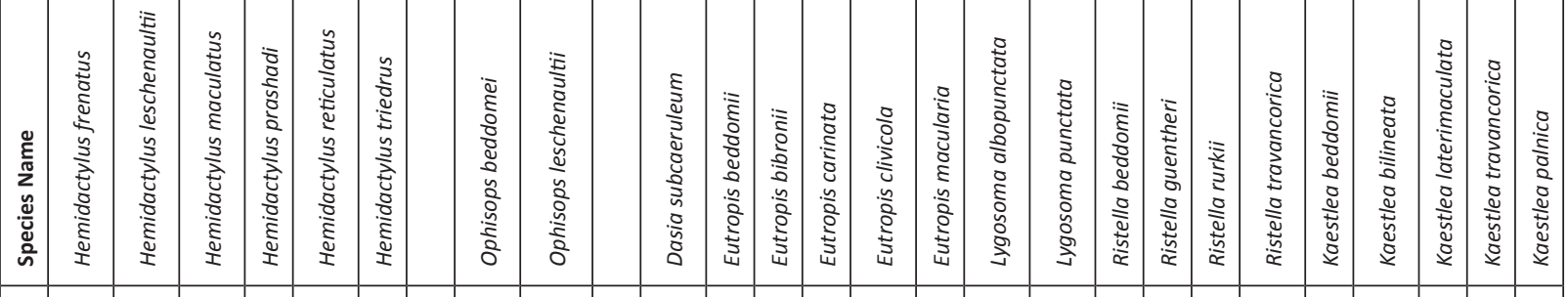

\begin{tabular}{|c|c|c|c|c|c|c|c|c|c|c|c|c|c|c|c|c|c|c|c|c|c|c|c|c|}
\hline 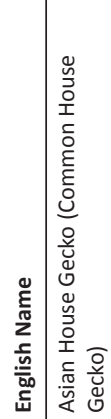 & 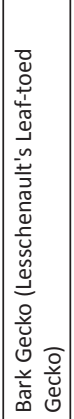 & 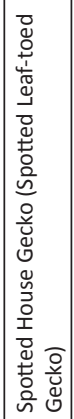 & 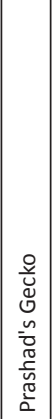 & 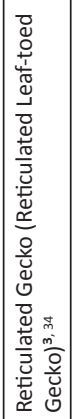 & 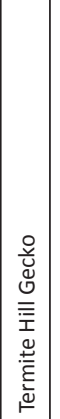 & 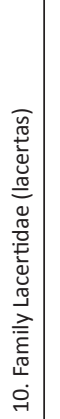 & 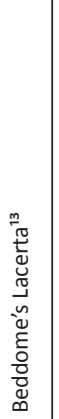 & 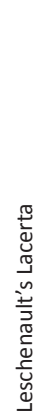 & 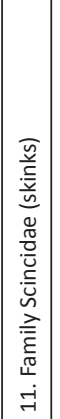 & 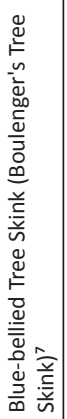 & 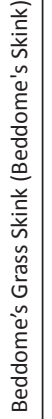 & 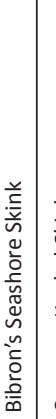 & 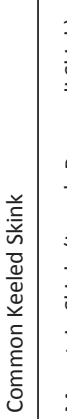 & 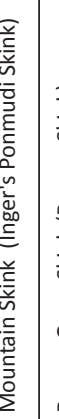 & 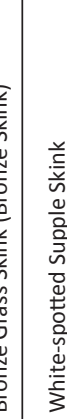 & 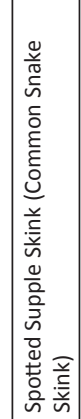 & 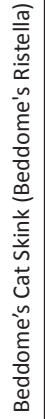 & 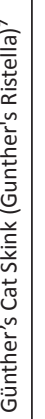 & & & & & & 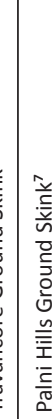 \\
\hline F & ケ & \& & $f$ & $\stackrel{\infty}{\text { 。 }}$ & g & & in & છี & & กี & ๓ & 出 & ถึ & in & $\stackrel{\infty}{n}$ & ถู & 8 & $\vec{\sigma}$ & & & & & $: 0$ & \\
\hline
\end{tabular}




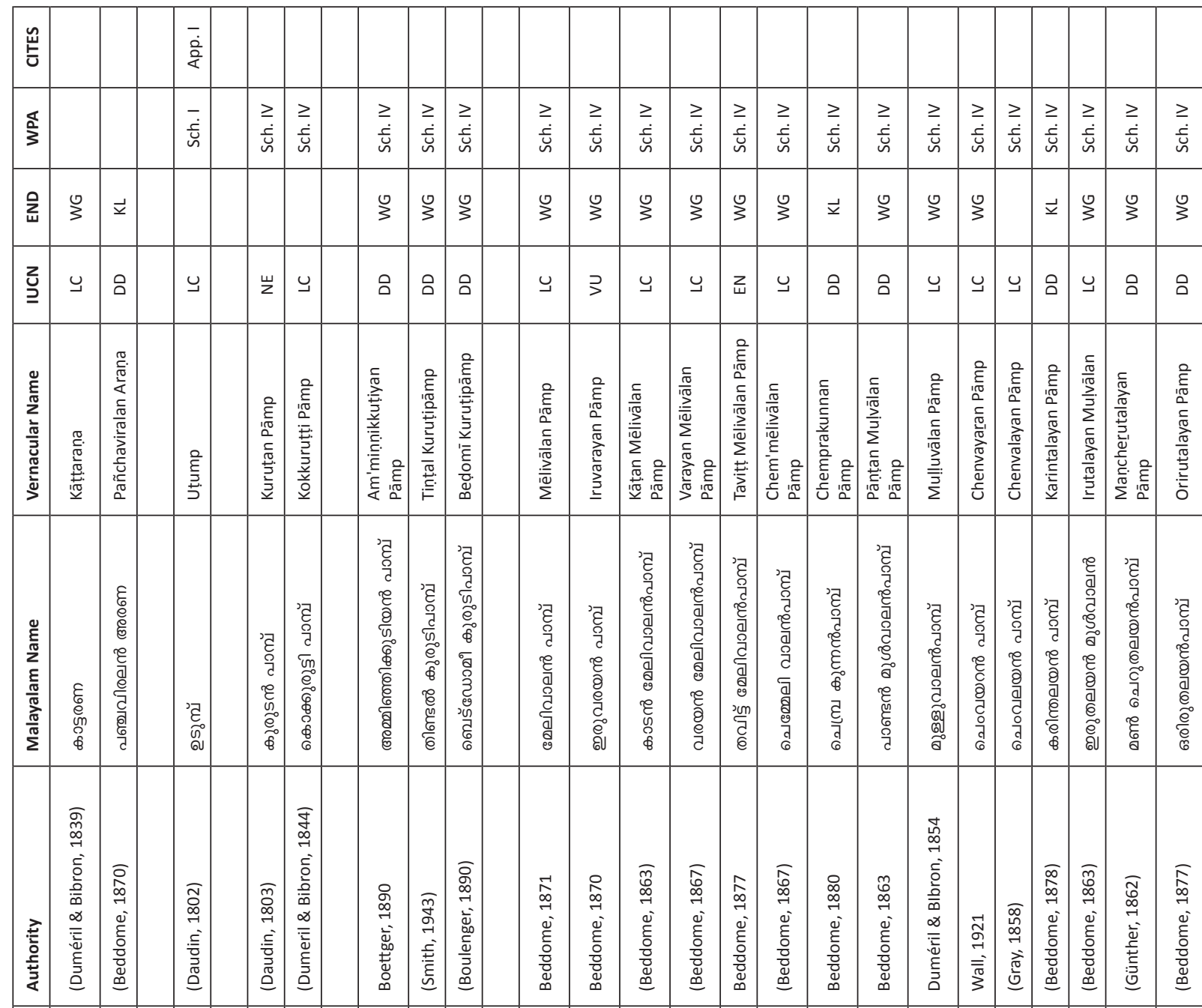

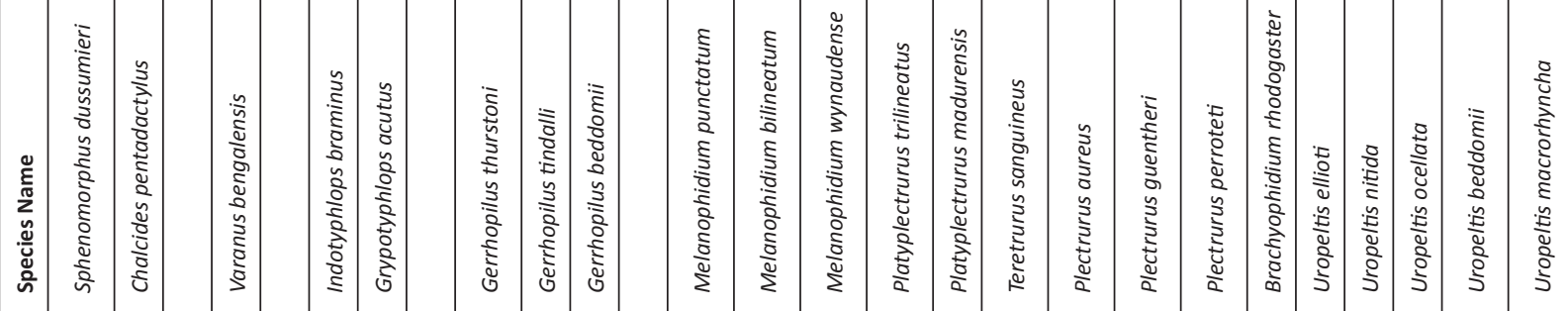

\begin{tabular}{|c|c|c|c|c|c|c|c|c|c|c|c|c|c|c|c|c|c|c|c|c|c|c|c|}
\hline 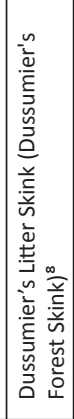 & 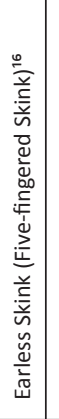 & 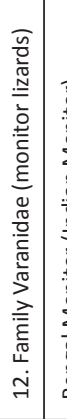 & 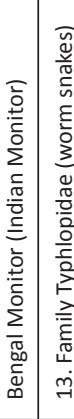 & 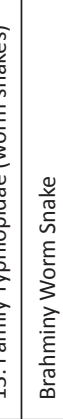 & 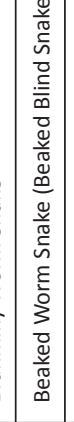 & 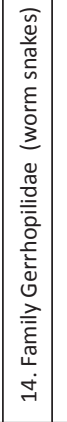 & 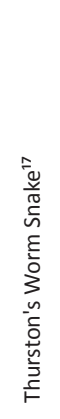 & 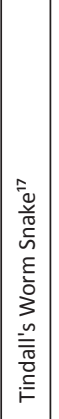 & 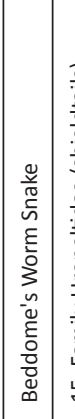 & 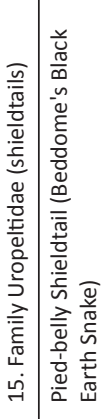 & 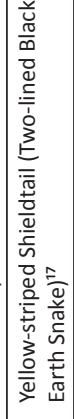 & 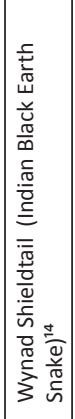 & 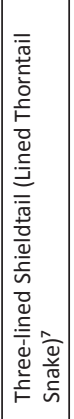 & 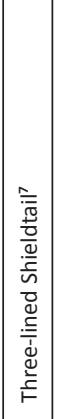 & 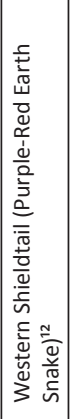 & 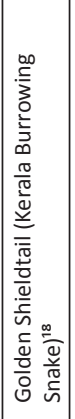 & 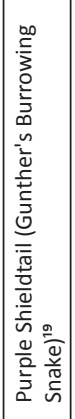 & 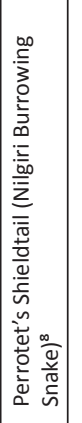 & 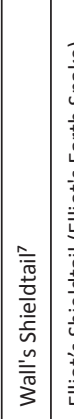 & 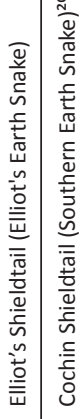 & 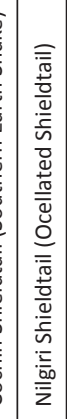 & 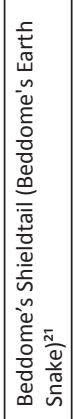 & 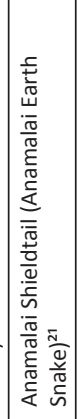 \\
\hline 8 & R & & ה & $\approx$ & N & & I & $\stackrel{n}{n}$ & 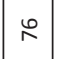 & 尺 & $\stackrel{\infty}{\wedge}$ & r & $\infty$ & $\vec{\infty}$ & $\infty$ & 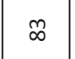 & $\Phi$ & 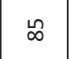 & $\begin{array}{lll}\infty & 0\end{array}$ & \begin{tabular}{c|c|c}
$\infty$ & $\infty$
\end{tabular} & $\infty$ & ๑ & ने \\
\hline
\end{tabular}




\begin{tabular}{|c|c|c|c|c|c|c|c|c|c|c|c|c|c|c|c|c|c|c|c|c|c|c|c|}
\hline 峞 & & & & & & & & & & & & & & & $\overline{\frac{0}{8}}$ & $\overline{\overline{\frac{i}{\alpha}}}$ & $\begin{array}{l}\overline{\bar{j}} \\
\frac{0}{\alpha}\end{array}$ & 产 & & & & & \\
\hline$\sum_{3}^{\frac{\Phi}{~}}$ & 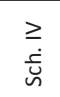 & $\begin{array}{l}\geq \\
\text { : } \\
\text { ¿ }\end{array}$ & $\begin{array}{l}\geq \\
\text { 离 }\end{array}$ & $\begin{array}{l}\geq \\
\text { 宫 }\end{array}$ & $\begin{array}{l}\geq \\
\geq \\
\dot{\bar{n}}\end{array}$ & $\begin{array}{l}\geq \\
\geq \\
\dot{\bar{s}}\end{array}$ & $\begin{array}{l}\geq \\
\geq \\
\dot{\bar{n}}\end{array}$ & $\begin{array}{l}\geq \\
\dot{\vec{L}} \\
\mathbf{w}\end{array}$ & 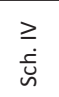 & $\begin{array}{l}\geq \\
\geq \\
\dot{\bar{n}} \\
\dot{n}\end{array}$ & $\begin{array}{l}\geq \\
\geq \\
\dot{\bar{s}}\end{array}$ & 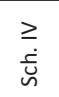 & 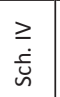 & $\begin{array}{l}\geq \\
\stackrel{\dot{j}}{\dot{m}}\end{array}$ & $\overline{\overline{\dot{c}}}$ & 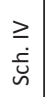 & $\begin{array}{l}\geq \\
\text { 它 } \\
w\end{array}$ & $\begin{array}{l}\geq \\
\geq \\
\dot{\bar{\omega}}\end{array}$ & $\begin{array}{l}\geq \\
\text { 离 }\end{array}$ & $\begin{array}{l}\geq \\
\dot{\vec{L}} \\
\bar{n}\end{array}$ & $\begin{array}{l}= \\
\bar{i} \\
\overrightarrow{\dot{\nu}}\end{array}$ & $\begin{array}{l}\geq \\
\geq \\
\dot{\bar{\omega}}\end{array}$ & $\begin{array}{l}\geq \\
\text { 忘 }\end{array}$ \\
\hline$\sum_{u}^{0}$ & $\stackrel{0}{3}$ & & $\coprod_{3}^{0}$ & $\stackrel{0}{3}$ & $\stackrel{0}{3}$ & $\stackrel{0}{3}$ & $\stackrel{0}{3}$ & $\stackrel{0}{3}$ & $\stackrel{0}{3}$ & $\stackrel{0}{3}$ & $\stackrel{0}{3}$ & $\varliminf_{3}^{0}$ & $\overrightarrow{\underline{v}}$ & $\stackrel{0}{3}$ & & & & & & & & & \\
\hline z & צ & u & צ & u & צ & ฉ & ฉ & 8 & ฉ & y & z & צ & ฉิ & z & u & $\underline{z}$ & w & $\underline{\mathbf{z}}$ & צ & $\frac{u}{z}$ & $\frac{w}{z}$ & $\underline{\mathrm{z}}$ & 崩 \\
\hline 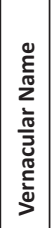 & 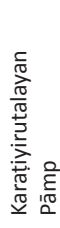 & 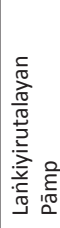 & 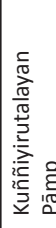 & 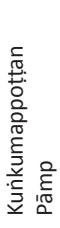 & 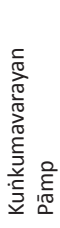 & 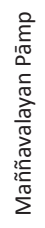 & 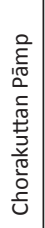 & 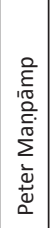 & 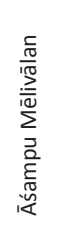 & 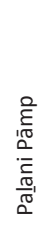 & 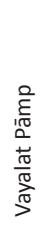 & 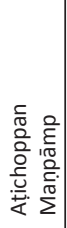 & 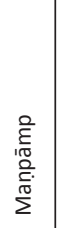 & 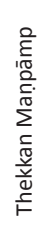 & 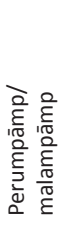 & 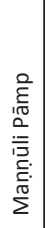 & 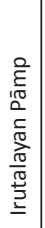 & 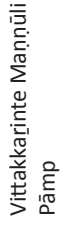 & 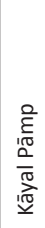 & 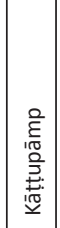 & 选 & 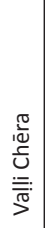 & 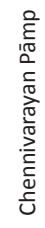 \\
\hline
\end{tabular}

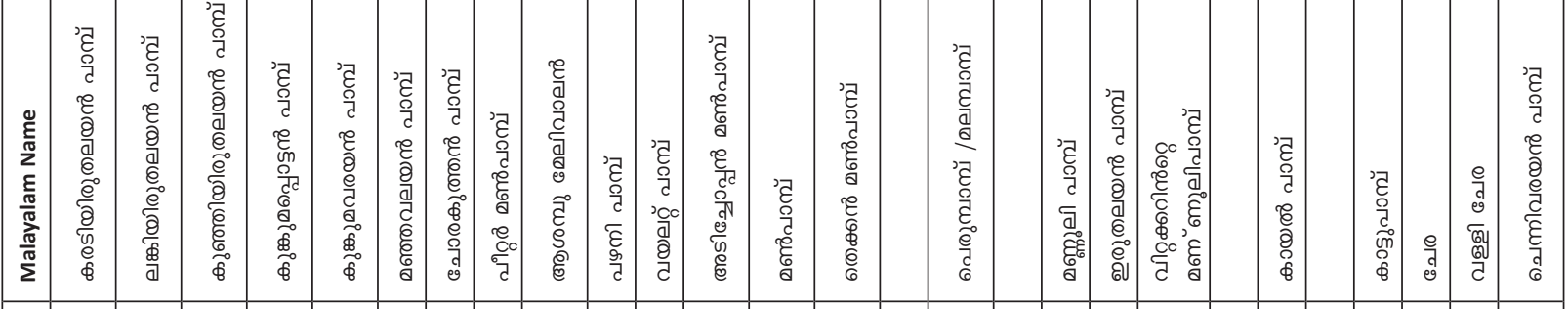

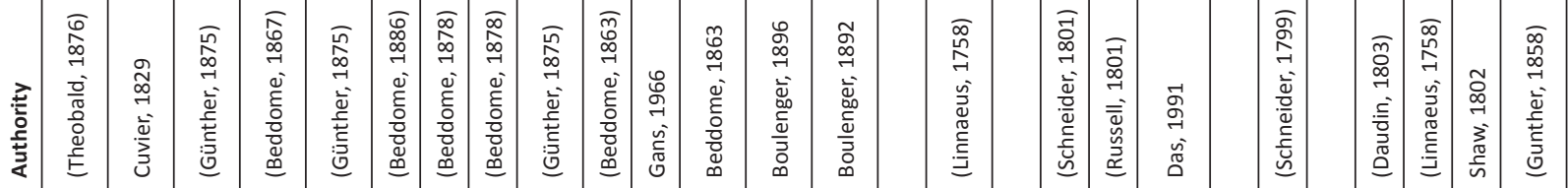

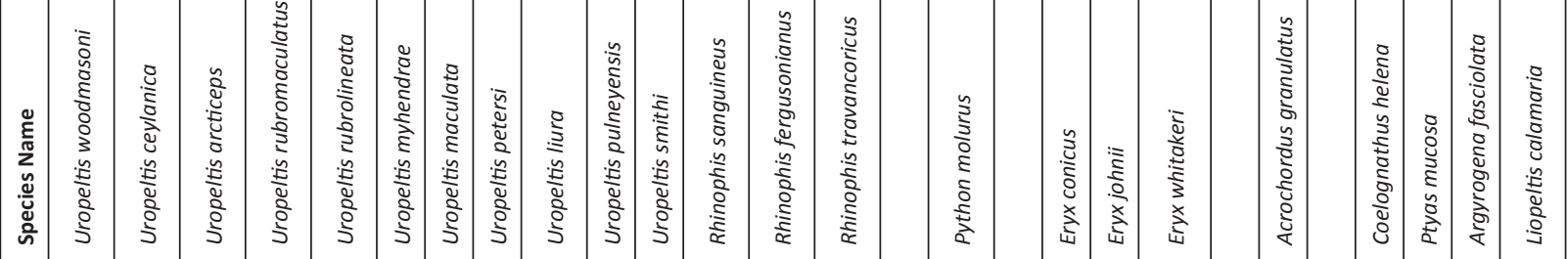

\begin{tabular}{|c|c|c|c|c|c|c|c|c|c|c|c|c|c|c|c|c|c|c|c|c|c|c|c|c|}
\hline 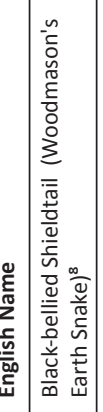 & 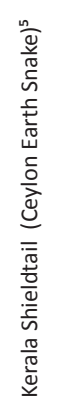 & 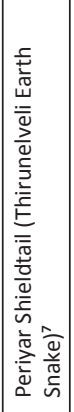 & 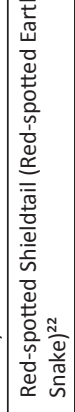 & 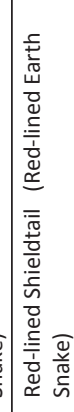 & 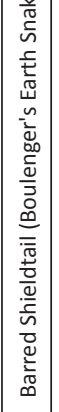 & 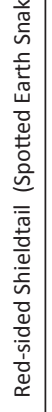 & 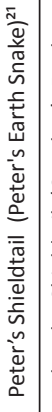 & 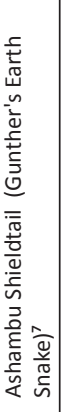 & 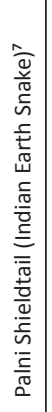 & 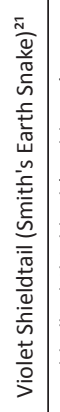 & 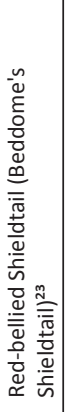 & 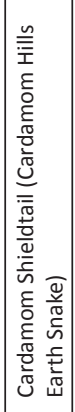 & 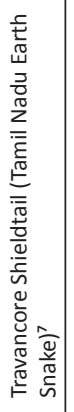 & 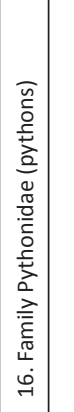 & 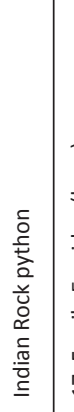 & 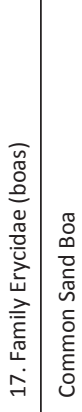 & 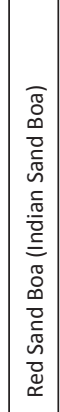 & 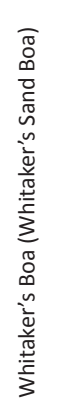 & 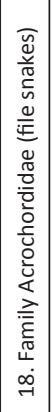 & 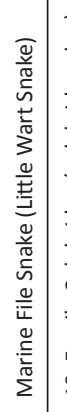 & 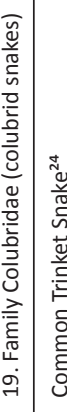 & 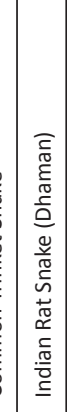 & 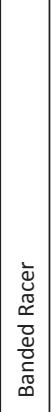 & 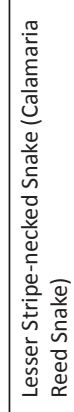 \\
\hline ส & n & ু & ณ & ஃ & ă & $\stackrel{\infty}{\circ}$ & Љ & \&్త & $\stackrel{\vec{\sigma}}{\vec{\sigma}}$ & స్త & $\stackrel{n}{a}$ & 声 & 号 & & : & of & $\stackrel{\infty}{\circ}$ & \& & & 의 & & $\exists$ & $\stackrel{m}{\exists}$ & \\
\hline
\end{tabular}




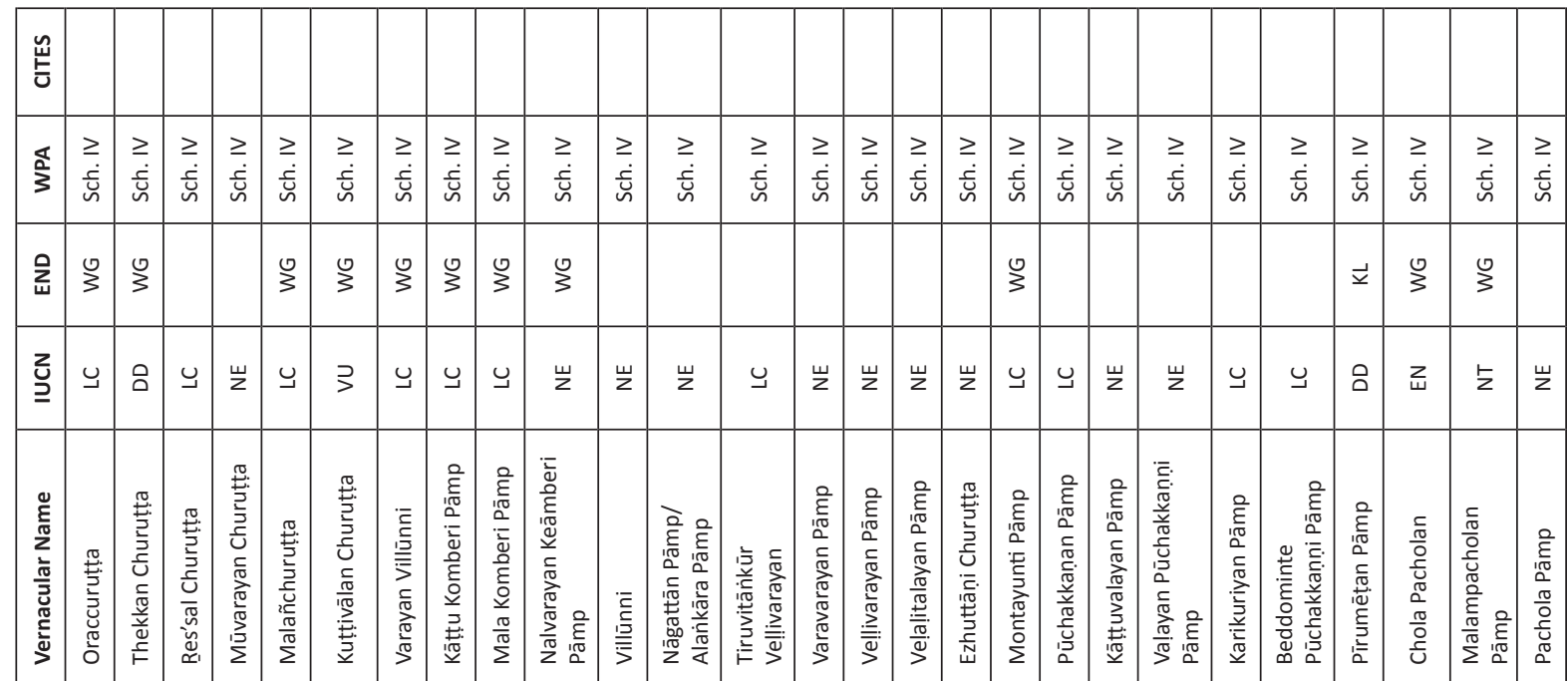

\begin{tabular}{|c|c|c|c|c|c|c|c|c|c|c|c|c|c|c|c|c|c|c|c|c|c|c|c|c|}
\hline & 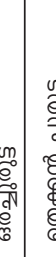 & 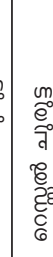 & 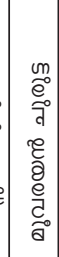 & 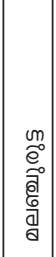 & 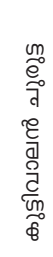 & $\begin{array}{l}\bar{\varepsilon} \\
\bar{a}: \\
\bar{c} \\
q \\
\bar{g} \\
\bar{e}\end{array}$ & 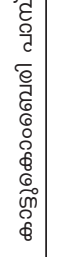 & 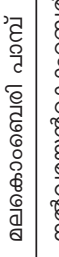 & 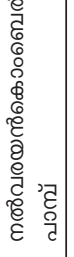 & \begin{tabular}{|c} 
\\
$\bar{\varepsilon}$ \\
$\overline{\tilde{a}}$ \\
$\bar{c}$ \\
$\bar{c}$
\end{tabular} & 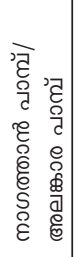 & 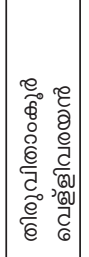 & 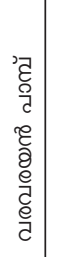 & 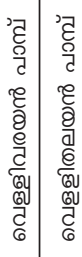 & 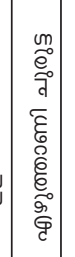 & 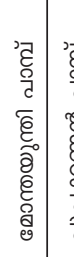 & 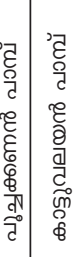 & 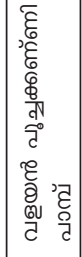 & 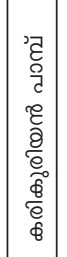 & 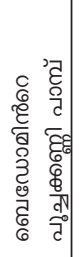 & 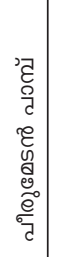 & 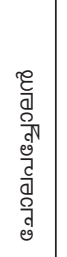 & 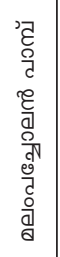 & \\
\hline & $\begin{array}{l}\infty \\
0 \\
0 \\
0 \\
0 \\
0 \\
0\end{array}$ & $\begin{array}{l}\text { M. } \\
\text { de }\end{array}$ & 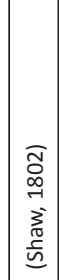 & 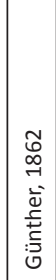 & 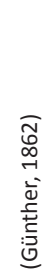 & 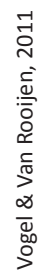 & 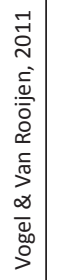 & 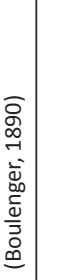 & 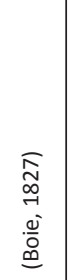 & 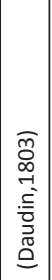 & 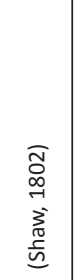 & 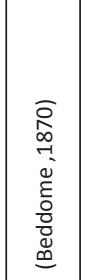 & 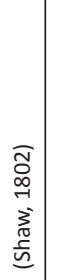 & 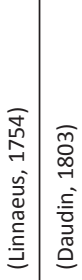 & 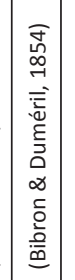 & 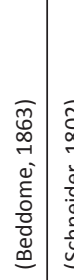 & 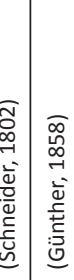 & 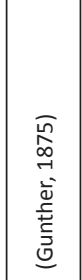 & 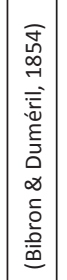 & 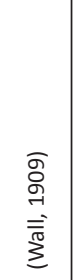 & 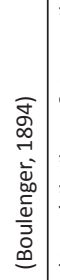 & 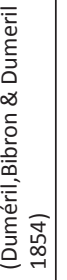 & 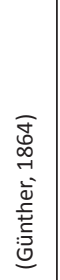 & \\
\hline
\end{tabular}

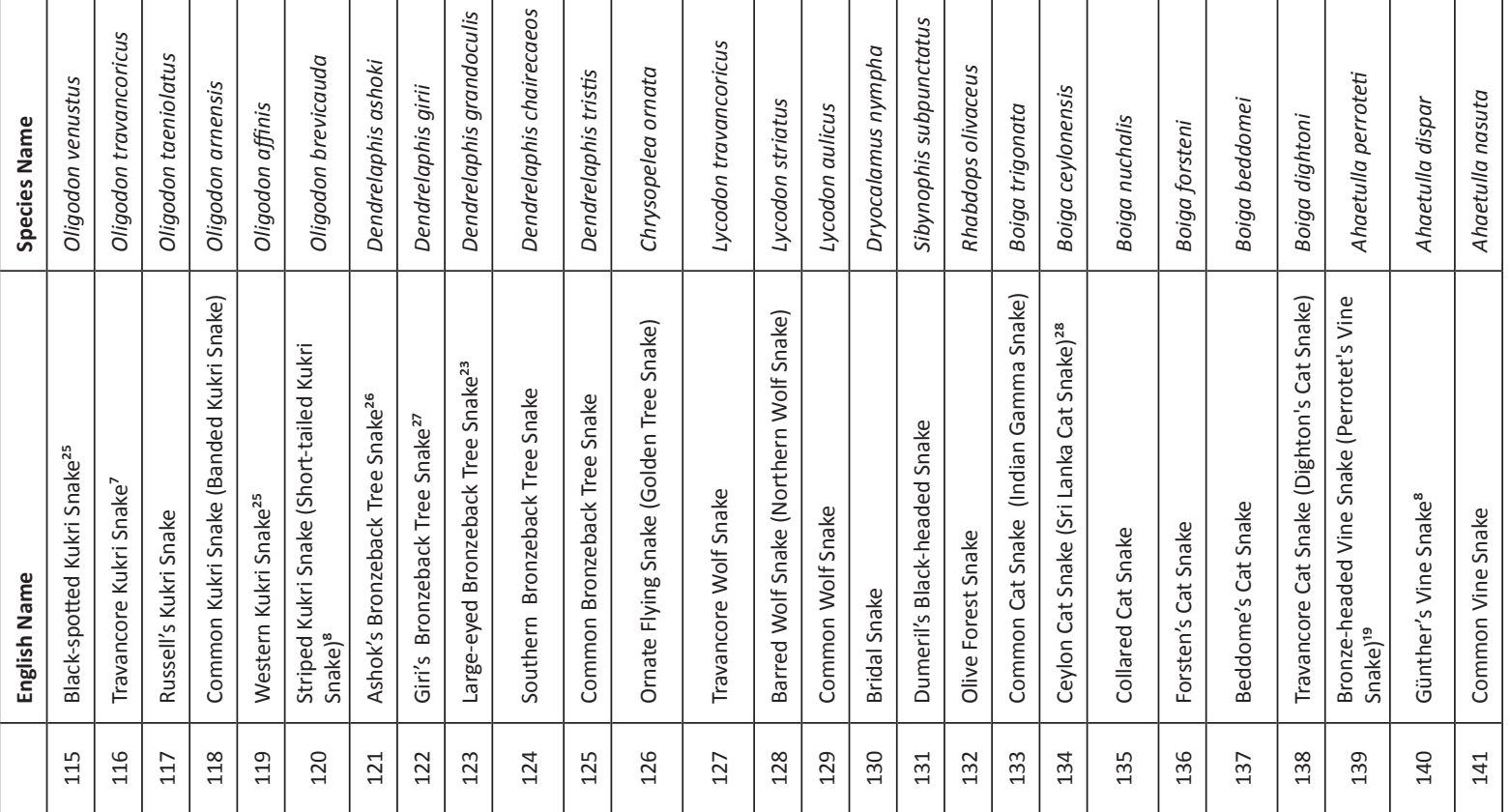




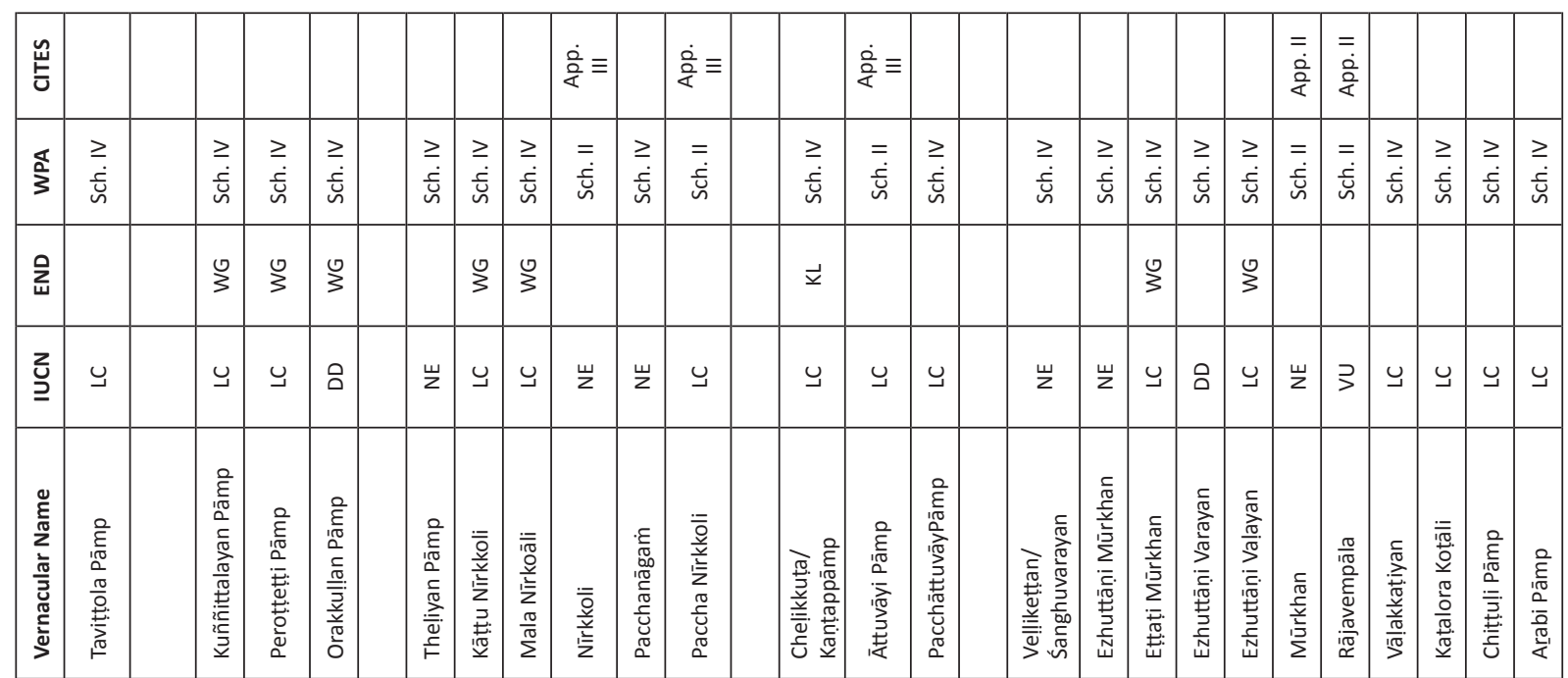

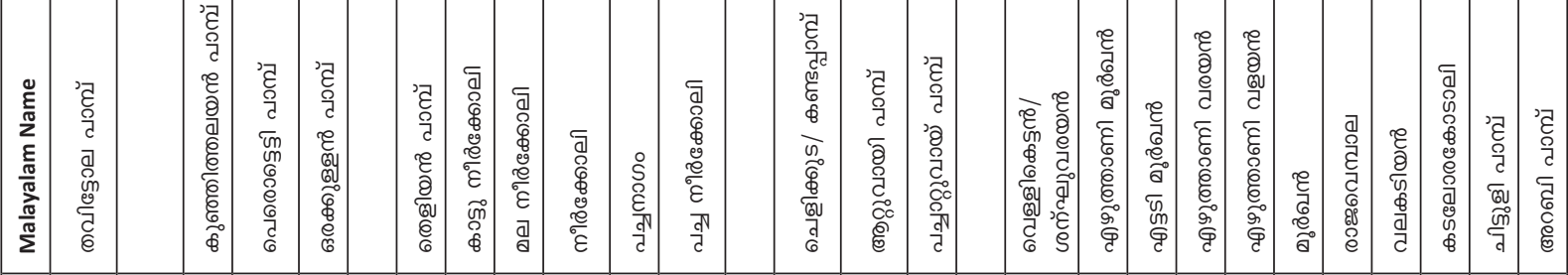

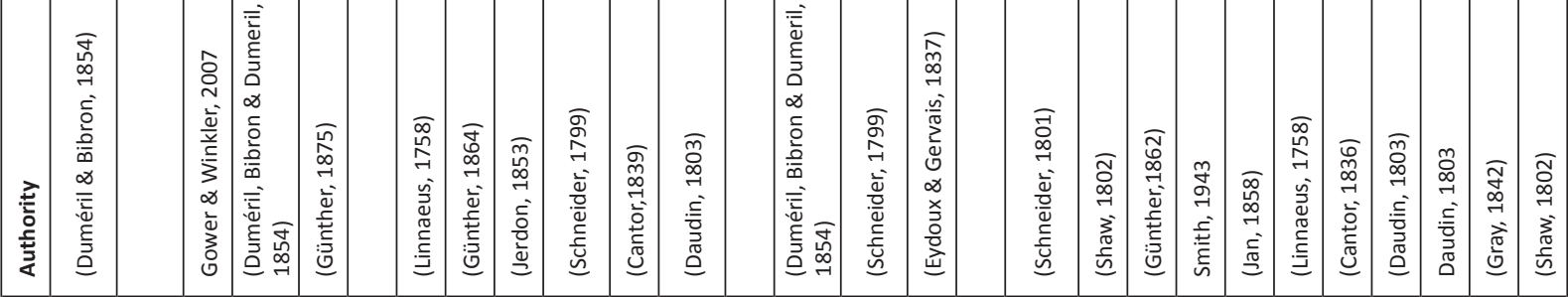

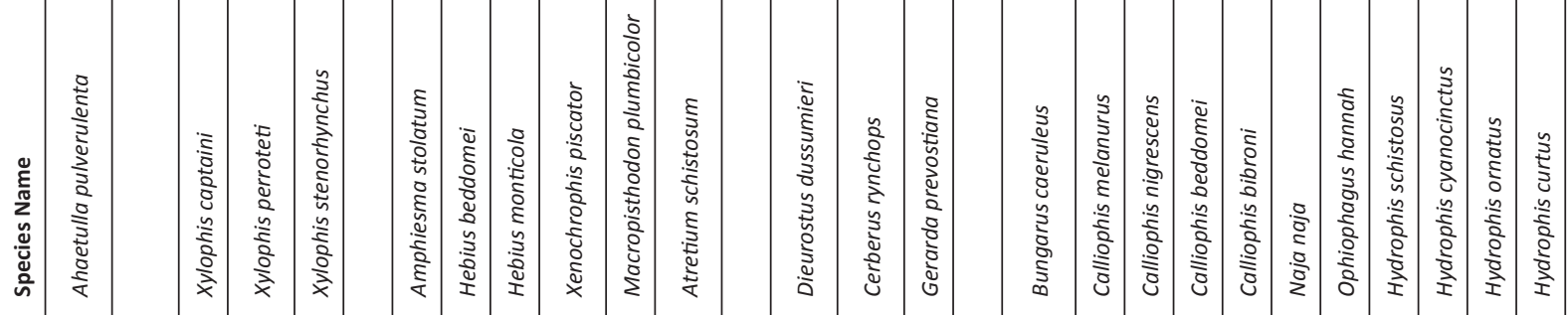

\begin{tabular}{|c|c|c|c|c|c|c|c|c|c|c|c|c|c|c|c|c|c|c|c|c|c|c|c|c|}
\hline 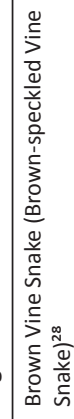 & 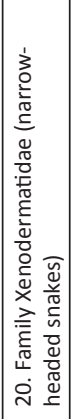 & 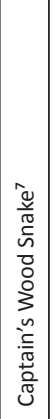 & 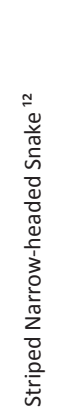 & 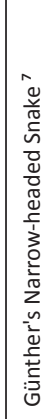 & 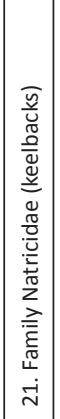 & 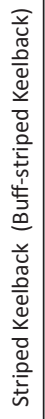 & 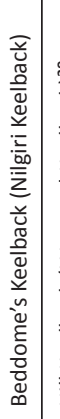 & 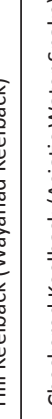 & . & 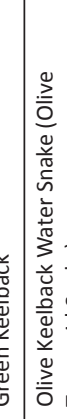 & 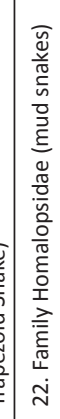 & 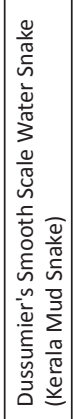 & 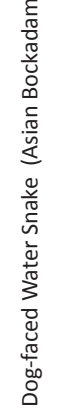 & 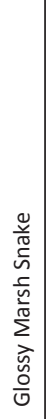 & 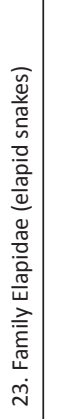 & 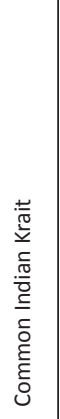 & 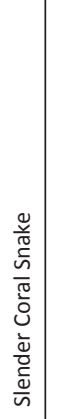 & 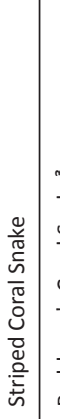 & 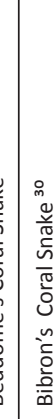 & 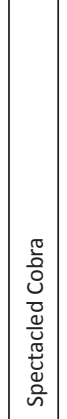 & 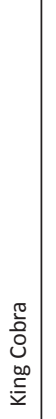 & 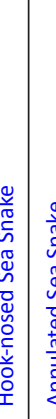 & & \\
\hline $\mathcal{I}$ & & & $\underset{J}{\mathbb{N}}$ & 导 & & $\underset{G}{g}$ & 寸 & & & & & 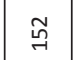 & 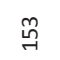 & 鴶 & & 男 & 号 & 今ి & & : & $\vec{\Xi}$ & & 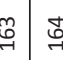 & \\
\hline
\end{tabular}




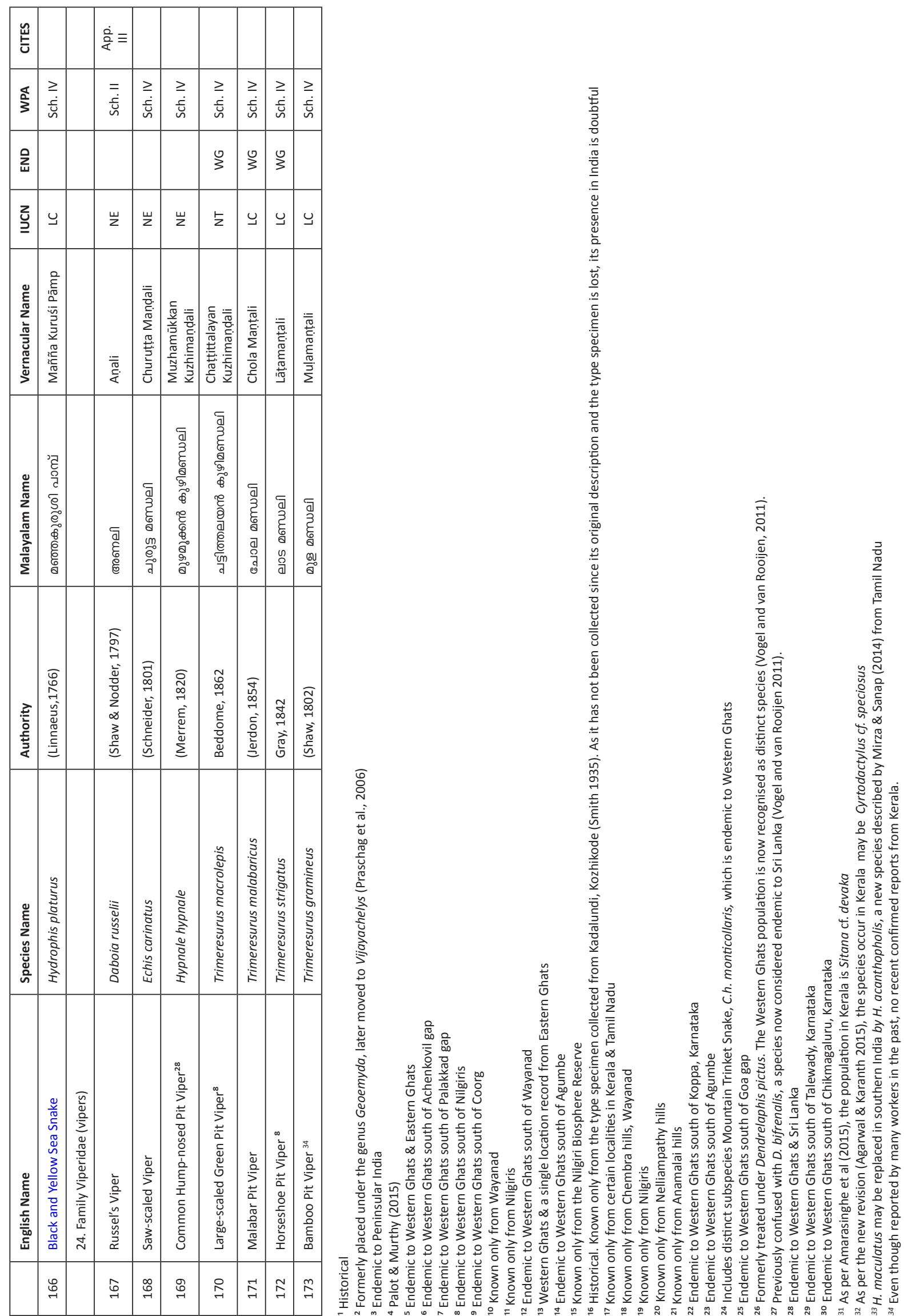


Scincidae), with the description of a new species from southern India. Zootaxa 3233: 37-51; http://dx.doi.org/10.11646/ zootaxa.3914.4.9

Inger, R.F., H.B. Shaffer, M. Koshy \& R. Bakde (1984). A report on the collection of amphibians and reptiles from Ponmudi, Kerala, south India. Journal of the Bombay Natural History Society 81: 406-427 and 551-570.

Inger, R.F., H. Marx \& M. Koshy (1984). An undescribed species of gekkonid lizard (Cnemaspis) from India with comments on the status of C. tropidogaster. Herpetologica 40(2): 149-154.

Inger, R.F., H.B. Shaffer, M. Koshy \& R. Bakde (1987). Ecologica structure of a herpetological assemblage in South India. Amphibia Reptilia 8: 189-202.

Jerdon, T.C. (1853). Catalogue of the Reptiles inhabiting the Peninsula of India. Journal of Asiatic Society of Bengal, pp. 462-79 and 52234.

Jins, V.J., S. Bhupathy \& M. Panigrahi (2014). New record of Beddome's Coral Snake, Calliophis beddomei Smith 1943 from the southern Western Ghats, India. Herpetology Notes 7: 555-557.

Joseph, T. \& P.S. Easa (1997). Additions to the Reptilian fauna of Silent Valley. Cobra.27: 31-33.

Manamendra-Arachchi, K., S. Batuwita \& R. Pethiyagoda (2007) A taxonomic revision of the Sri Lankan day-Geckos (Reptilia: Gekkonidae: Cnemaspis), with description of new species from Sri Lanka and southern India. Zeylanica 7(1): 9-122.

Mirza, Z.A \& R.V. Sanap (2014). A new cryptic species of Gecko of the genus Hemidactylus Oken, 1817 (Reptilia: Gekkonidae) from southern India. Taprobanica 6(1): 12-20.

Mirza, Z.A., S. Pal, H.S. Bhosale \& R.V. Sanap (2014). A new species of gecko of the genus Cnemaspis Strauch, 1887 from the Western Ghats, India. Zootaxa 3815(4): 494-506; http://dx.doi. org/10.11646/zootaxa.3815.4.2

Mirza, Z.A., R.V. Sanap, D. Raju, A. Gawai \& P. Ghadekar (2014). A new species of lizard of the genus Eublepharis (Squamata: Eublepharidae) from India. Phyllomedusa 13(2): 75-90; http:// dx.doi.org/10.11606/issn.2316-9079.v13i2p75-90

Molur, S. \& S. Walker (1998) (eds.). Report of BCCP CAMP on Reptiles of India. Zoo Outreach Organisation, Coimbatore, India, 65pp.

Murthy, T.S.N. (1981). Reptiles of the Silent Valley and the New Amarambalam area, Kerala. Snake 13: 452.

Murthy, T.S.N. (1985). A field guide to the Lizards of Western Ghats. Records of Zoological Survey of India Occasional Paper No.72, 51pp.

Palot, M.J. (2013). Reptiles, pp. 179-210. In: Venkatraman, K., A Chattopadhyay \& K.A. Subramanian (eds.). Endemic Animals of India (Vertebrates): $1-235+26$ pls (Published by the Director, Zoological Survey of India, Kolkata).

Palot, M.J. \& C. Radhakrishnan (2002). Herpetofauna of Madayipara Hill, Kannur District, Kerala. Cobra 48: 3-4.

Palot, M.J. \& C. Radhakrishnan (2003). Checklist of snakes of Kerala with their Malayalam vernacular names. Reptile Rap 5: 3-6.

Palot, M.J. \& C. Radhakrishnan (2004). Status and distribution of Turtle fauna (Testudines: Reptilia) in the Malabar part of Kerala, India. Records of Zoological Survey of India 102 (part 1-2): 27-39.

Palot, M.J. \& C. Radhakrishnan (2011). An updated checklist of reptiles of Kerala. Malabar Trogon 9(1\&2): 24-30.

Palot, M.J. \& B.H.C. K. Murthy. (2015). First record of the Narrowheaded Softshell Turtle, Chitra indica (Gray 1830) from Kerala, India. Malabar Trogon 13(1): 31-32.

Radhakrishnan, C. (1996). Survey of Reptilian fauna of Parambikulam Wildlife Sanctuary, Kerala. Cobra 24: 7-12.

Radhakrishnan, C. (1997). Reptiles. In: Natural Resources of Kerala. (Eds.) WWF- India, Kerala Chapter, Thiruvananthapuram.

Rajendran, M.V. (1985). Studies in uropeltid snakes. Madurai (India): Publication Division, Madurai Kamaraj University, VI (unnumbered) +132pp.
Sharma, R.C. (1998). Fauna of India and the Adjacent Countries Reptilia (Testudines and Crocodilia). Vol. I. Published by the Director, Zoological Survey of India, Kolkata, 196pp.

Sharma, R.C. (2002). The Fauna of India and the Adjacent Countries Reptilia (Sauria). Vol. II. Published by the Director, Zoological Survey of India, Kolkata, 430pp.

Sharma, R.C. (2007). The Fauna of India and the Adjacent Countries Reptilia (Serpentes). Vol. 111. Published by the Director, Zoological Survey of India, Kolkata, 410pp.

Smith, M.A. (1931). The Fauna of British India Including Ceylon and Burma, Including the whole of Indo-Chinese Sub-Region: Vol.1. Loricata, Testudines. Taylor and Francis, London.

Smith, M.A. (1935). The Fauna of British India Including Ceylon and Burma, Including the whole of Indo-Chinese Sub-Region: Vol II. Sauria. Taylor and Francis, London.

Smith, M.A. (1943). The Fauna of British India Including Ceylon and Burma, Including the Whole of Indo-Chinese Sub-Region: Vol. III. Serpentes. Taylor and Francis, London.

Smith, E.N., H. Ogale, V. Deepak \& V.B. Giri (2012). A new species of coral snake of the genus Calliophis (Squamata: Elapidae) from the west coast of peninsular India. Zootaxa 3437: 51-68

Thomas, J., J. Sabu \& P.S. Easa (1997). Status and distribution of reptiles in Wayanad, Kerala. Cobra 28: 25-30.

Srinivasulu, C., B. Srinivasulu \& S. Molur (Compilers) (2014). The Status and Distribution of Reptiles in the Western Ghats, India. Conservation Assessment and Management Plan (CAMP). Wildlife Information Liaison Development Society, Coimbatore, Tamil Nadu.

Thomas, J. \& P.S. Easa (1997). Reptile Fauna of Peechi-Vazhani Wildlife Sanctuary. Cobra 29: 14-18.

Tikader, B.K. \& R.C. Sharma (1992). Handbook of Indian Reptiles. Zoological Survey of India, Calcutta, 250pp+42pls.

Uetz, P. \& J. Hosek (eds.) (2014). The Reptile Database. http://www. Reptile database.org. accessed on 30 September 2014.

van Rooijen, J. \& G. Vogel (2009). A multivariate investigation into the population systematics of Dendrelaphis tristis (Daudin, 1803) and Dendrelaphis schokari (Kuhl, 1820): revalidation of Dendrophis chairecacos Boie, 1827 (Serpentes: Colubridae). The Herpetological Journal 19: 193-200.

Vasudevan, K. (ed.). (2009). Freshwater Turtles and Tortoises of India. ENVIS Bulletin: Wildlife and Protected Areas. Vol. 12 (1). Wildlife Institute of India, Dehradun, India, 177pp.

Venugopal, P.D. (2010). An updated and annotated list of Indian Lizards (Reptilia: Sauria) based on a review of distribution records and checklist of Indian reptiles. Journal of Threatened Taxa 2(3): 725-738; http://dx.doi.org/10.11609/JoTT.02083.725-38

Vogel, G. \& J. van Rooijen (2011). Contributions to a Review of the Dendrelaphis pictus (Gmelin, 1789) Complex (Serpentes: Colubridae) - 3. The Indian Forms, with the Description of a New Species from the Western Ghats. Journal of Herpetology 45(1): 100110; http://dx.doi.org/10.11646/zootaxa.3637.2.6

Vogel, G. \& S.R. Ganesh (2013). A new species of cat snake (Reptilia: Serpentes: Colubridae: Boiga) from dry forests of eastern peninsular India. Zootaxa 3637(2): 158-168.

Wall, F. (1905). Notes on snakes collected in Cannanore from 5 November 1903 to 5 August 1904. Journal of the Bombay Natural History Society 16: 292-312.

Wall, F. (1918). Notes on a collection of snakes made in the Nilgiri Hills and the adjacent Wayanad. Journal of the Bombay Natural History Society 6: 152-156.

Whitaker, R. \& A. Captain (2004). Snakes of India. The Field Guide. Draco Books. Chengalpattu, Tamil Nadu, xiv+479, pls, text-figs.

Zacharias, V.J. (1997). Reptiles of Periyar Tiger Reserve. Journal of the Bombay Natural History Society 94: 575-579. 


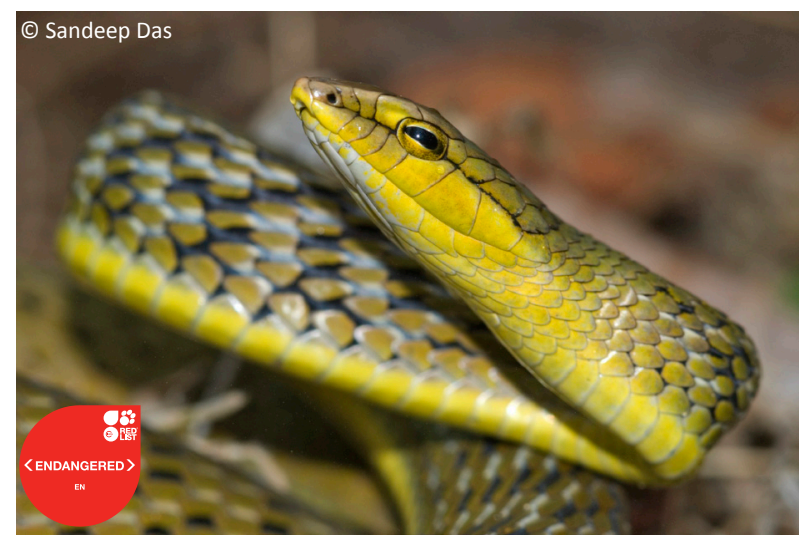

Image 1. Ahaetulla perroteti

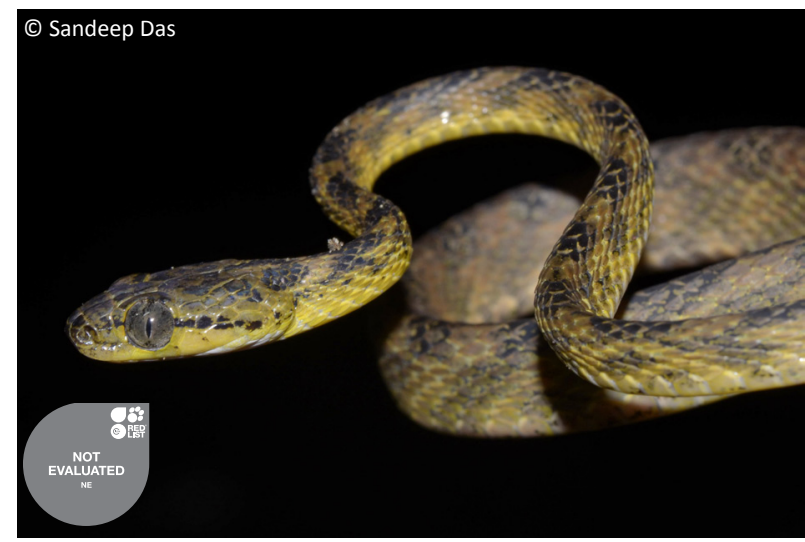

Image 3. Boiga ceylonensis

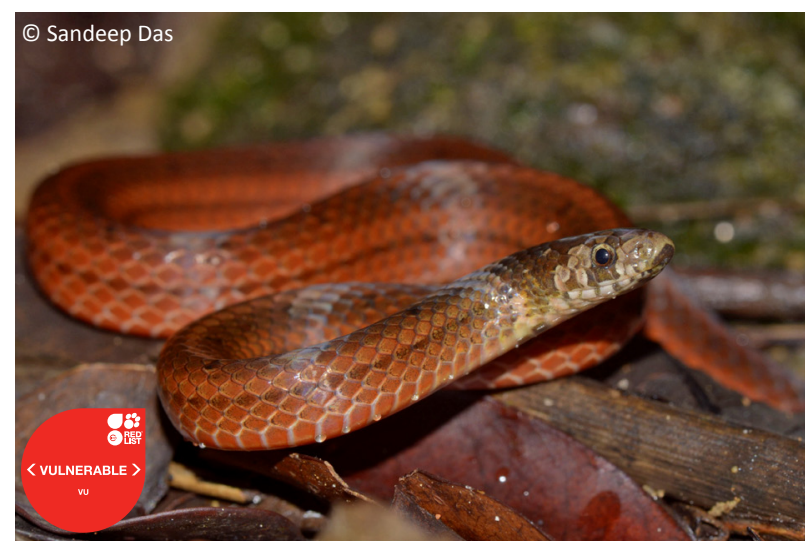

Image 5. Oligodon brevicauda

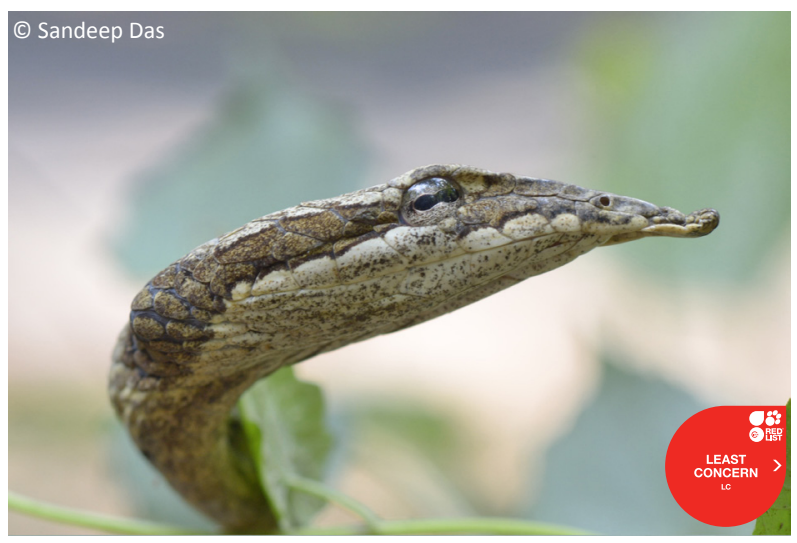

Image 2. Ahaetulla pulverulenta

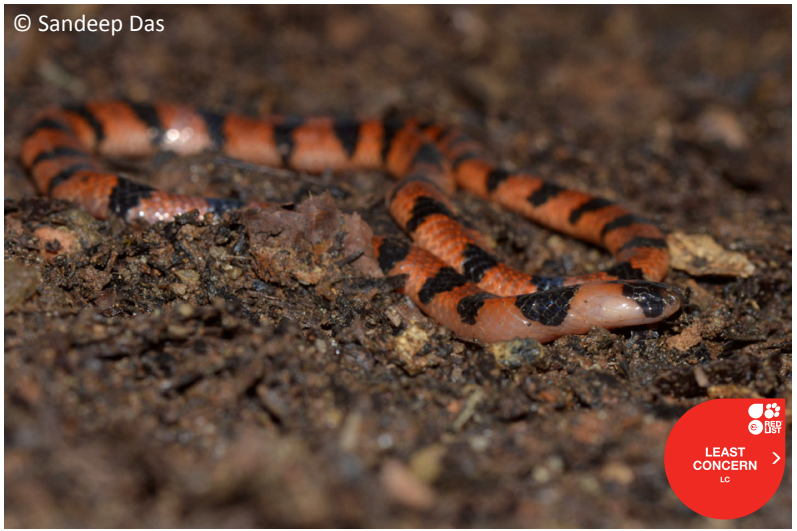

Image 4. Calliophis bibroni

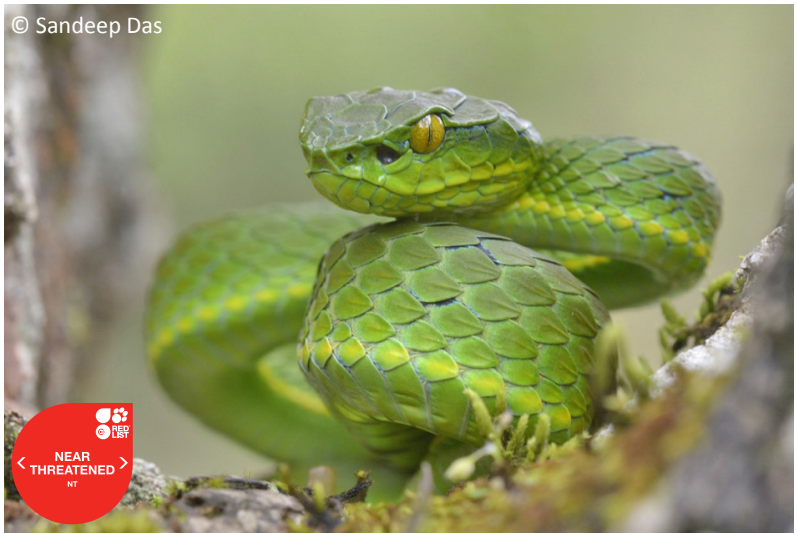

Image 6. Trimeresurus macrolepis 


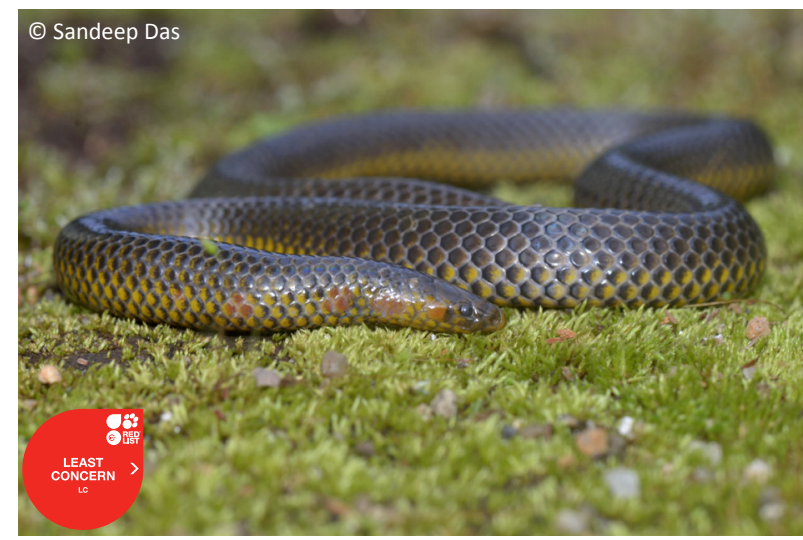

Image 7. Uropeltis rubromaculatus

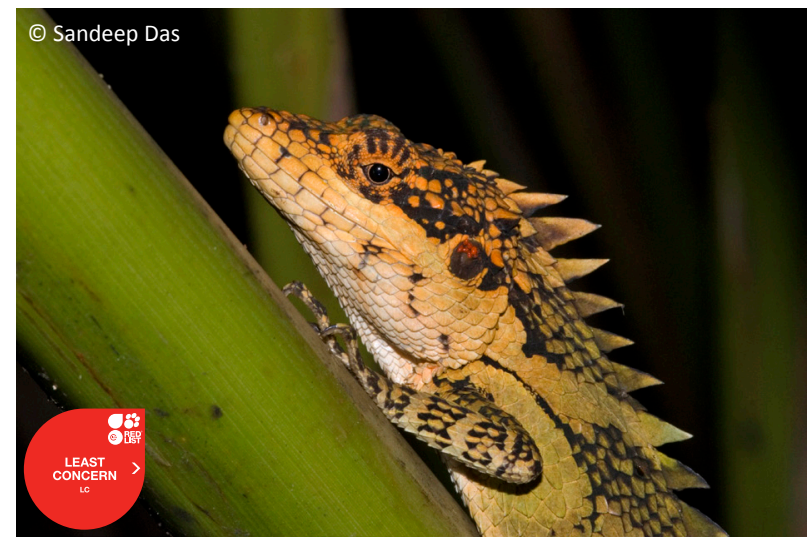

Image 9. Salea anamallayana

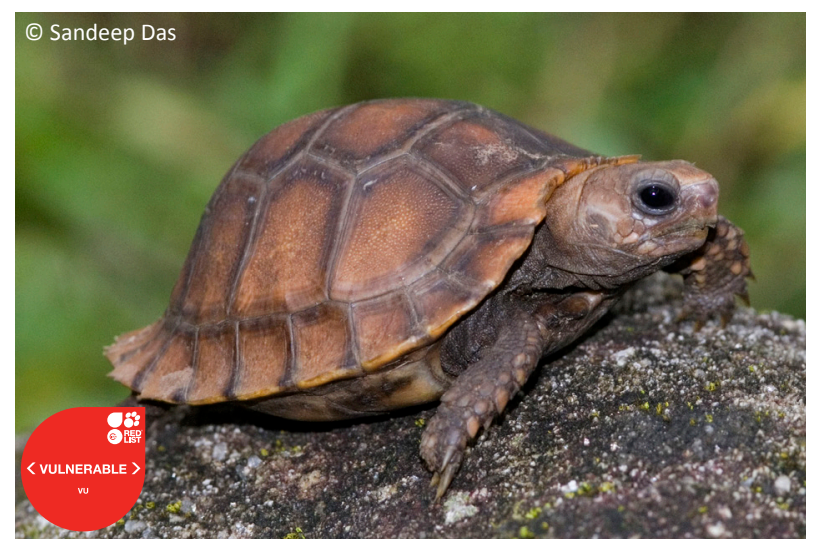

Image 11. Indotestudo travancorica

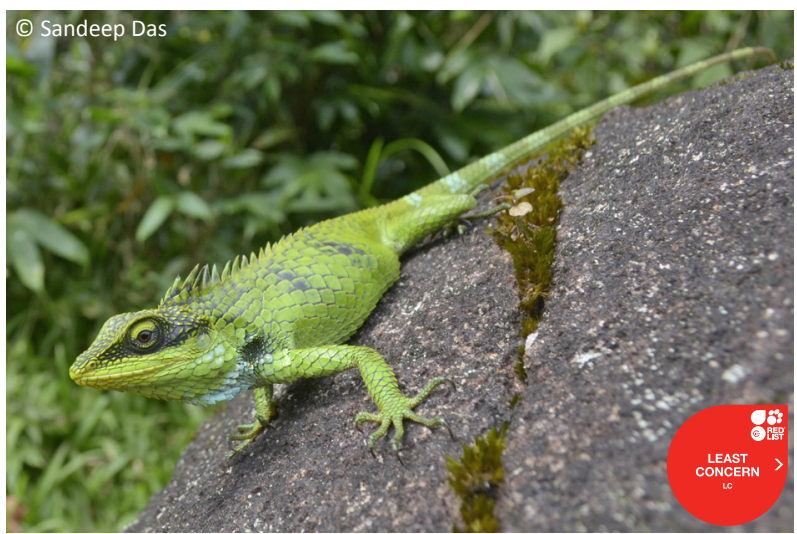

Image 8. Calotes grandisquamis

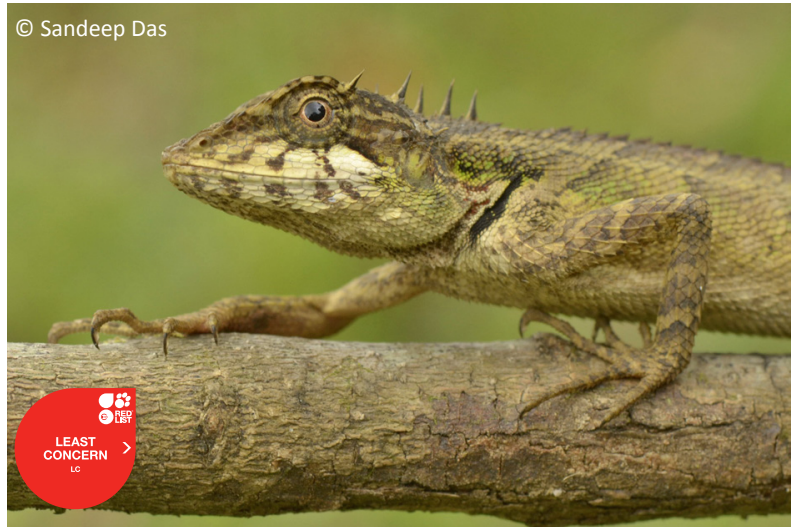

Image 10. Calotes ellioti

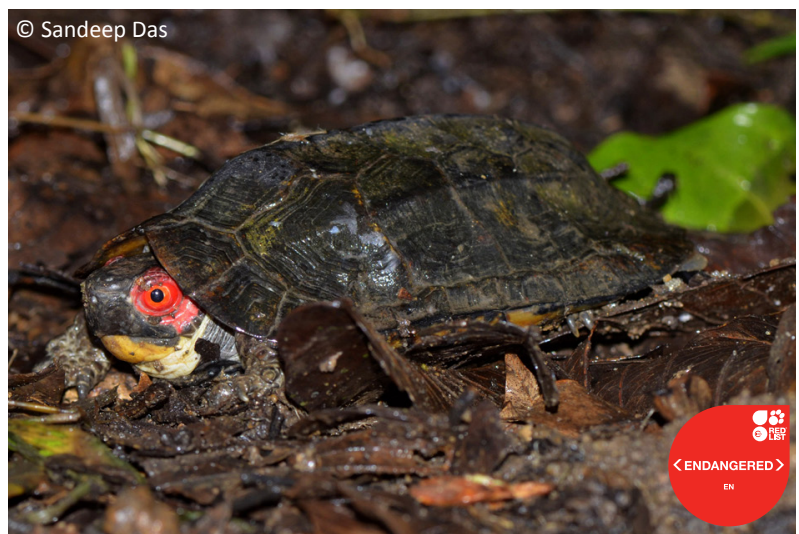

Image 12. Vijayachelys silvatica

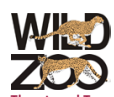

